ARTICLE

Received 14 Jun 2016 | Accepted 26 Apr 2017 | Published 9 Jun $2017 \quad$ DOl: 10.1038/ncomms15754

\title{
Constriction of the mitochondrial inner compartment is a priming event for mitochondrial division
}

Bongki Cho',2, Hyo Min Cho', Youhwa Jo', Hee Dae Kim³, Myungjae Song ${ }^{4}$, Cheil Moon², Hyongbum Kim, Kyungjin Kim ${ }^{2,5}$, Hiromi Sesaki ${ }^{6}$, Im Joo Rhyu ${ }^{1}$, Hyun Kim$^{1} \&$ Woong Sun ${ }^{1}$

Mitochondrial division is critical for the maintenance and regulation of mitochondrial function, quality and distribution. This process is controlled by cytosolic actin-based constriction machinery and dynamin-related protein 1 (Drp1) on mitochondrial outer membrane (OMM). Although mitochondrial physiology, including oxidative phosphorylation, is also important for efficient mitochondrial division, morphological alterations of the mitochondrial inner-membrane (IMM) have not been clearly elucidated. Here we report spontaneous and repetitive constriction of mitochondrial inner compartment (CoMIC) associated with subsequent division in neurons. Although CoMIC is potentiated by inhibition of Drp1 and occurs at the potential division spots contacting the endoplasmic reticulum, it appears on IMM independently of OMM. Intra-mitochondrial influx of $\mathrm{Ca}^{2}+$ induces and potentiates COMIC, and leads to $\mathrm{K}^{+}$-mediated mitochondrial bulging and depolarization. Synergistically, optic atrophy 1 (Opa1) also regulates CoMIC via controlling Mic60-mediated OMM-IMM tethering. Therefore, we propose that CoMIC is a priming event for efficient mitochondrial division.

\footnotetext{
${ }^{1}$ Department of Anatomy, Korea University College of Medicine, 145 Anam-ro, Seongbuk-gu, Seoul 02841, Republic of Korea. ${ }^{2}$ Department of Brain \& Cognitive Sciences, Daegu Gyeongbuk Institute of Science and Technology, 333 Techno Jungang-daero, Hyeonpung-myeon, Dalseong-gun, Daegu 42988, Republic of Korea. ${ }^{3}$ Department of Biological Sciences, Seoul National University, Seoul 08826, Republic of Korea. ${ }^{4}$ Department of Pharmacology, Yonsei University College of Medicine, 50-1 Yonsei-ro, Seodaemoon-gu, Seoul 03722, Republic of Korea. ${ }^{5}$ Korea Brain Research Institute, 61 Choeomdan-Ro, DongGu, Daegu 41068, Republic of Korea. ${ }^{6}$ Department of Cell Biology, Johns Hopkins University School of Medicine, Baltimore, Maryland 21205, USA. Correspondence and requests for materials should be addressed to W.S. (email: woongsun@korea.ac.kr).
} 
M itochondria continuously undergo fusion and division to regulate proper function, quality and distribution in response to changing cellular environments ${ }^{1}$. According to the endosymbiosis theory, the outer membrane (OMM) and inner membrane (IMM) of mitochondria have different chemical and molecular properties ${ }^{2}$. Thus, regulation of mitochondrial morphology requires the coordination of distinct machineries for OMM and $\mathrm{IMM}^{3}$. In mammalian cells, mitochondrial fusion is mediated by two sequential steps: OMM fusion by mitofusin 1/2 (Mfn1/2), and IMM fusion by optic atrophy 1 (Opa1). In contrast, mitochondrial division is known to be solely driven by dynamin-related protein 1 (Drp1). Drp1 translocates from the cytosol to OMM receptors including Fis1, Mff and MiD49/51 (ref. 4), and forms spiral-like structure by oligomerization ${ }^{5}$. Subsequently, this spiral structure constricts and divides a mitochondrion. However, structural biological studies have revealed that the Drp1 spiral is not sufficient for the initiation of mitochondrial constriction because mitochondrial diameter $(0.5-1.0 \mu \mathrm{m})$ is much thicker than the Drp1 spiral $(\sim 100 \mathrm{~nm})^{5,6}$, implying the requirement for an initial mitochondrial constriction step before Drp1 action. Recent reports have revealed that actin filaments promote initial mitochondrial constriction through direct contact of mitochondria with the endoplasmic reticulum $(\mathrm{ER})^{7,8}$, and dynamin 2 collaborates with Drp1 for finalization of mitochondrial division ${ }^{9}$. This mode of action is similar to endocytosis mediated by dynamin ${ }^{10}$, and recent reports have demonstrated that Drp1 is involved in endocytosis as well as mitochondrial division ${ }^{11,12}$. These findings suggest that Drp1dependent mitochondrial division evolutionarily originated from cytosolic vesicle-scissoring machineries ${ }^{2}$.

Endosymbiosis theory proposes that the IMM is evolutionarily derived from a prokaryote-like endosymbiont, while the OMM is derived from the plasma membrane ${ }^{2}$. Prokaryotic division is executed by FtsZ, a tubulin-like protein, forming intracellular Z-ring ${ }^{2}$. Consistently, primitive eukaryotes, such as red algae, have mitochondrial orthologues of FtsZ, and they exhibit a Z-ring during IMM constriction in the mitochondrial matrix before Drp1-mediated ring formation ${ }^{13}$. This information implies that mitochondria require another step in constriction of the IMM before the activation of cytosolic Drp1. However, neither FtsZlike proteins nor Z-ring structures have been identified in mammals ${ }^{2}$. Nonetheless, constriction or division of the IMM in the absence of Drp1 has been observed in yeast ${ }^{14}$, C. elegans ${ }^{15}$ and mouse fibroblasts ${ }^{16,17}$. Also, electron microscopy studies have shown that the IMM can divide in the absence of OMM division ${ }^{18}$. Although these reports support the notion that OMMindependent intra-mitochondrial event(s) may exist, its presence in mammalian cells and the underlying mechanisms have not been elucidated.

Numerous light-scattering experiments using isolated mitochondria have shown that intra-mitochondrial homoeostasis of cations, such as $\mathrm{Ca}^{2+}, \mathrm{K}^{+}$and $\mathrm{Na}^{+}$, is important for the regulation of mitochondrial volume, causing changes in mitochondrial function ${ }^{19}$. In particular, $\mathrm{K}^{+}$influx into the mitochondria induces swelling or bulging via osmosis. In addition, mitochondrial dysfunction by mitochondrial depolarization and $\mathrm{Ca}^{2+}$ overload induces Drp1-dependent mitochondrial fragmentation, which contributes to mitophagy or apoptosis ${ }^{20}$. In contrast, metabolically active mitochondria are elongated and are not readily fragmented by intracellular stressors, which is the result of enhanced fusion with Mfn1/2 and Opal and decreased activity of Drp1 (refs 21,22). Recent papers have reported that elongated mitochondria transiently form bead-on-a-string shapes with fluctuations in intramitochondrial metabolic status, membrane potential, ROS production and $\mathrm{Ca}^{2+}$ influx ${ }^{22-25}$. However, the significance of these morphological changes and their possible association with mitochondrial division has not yet been addressed.

In this study, we report that the transient changes in mitochondrial morphology to bead-on-a-string shape are primarily caused by intra-mitochondrial morphological alterations, referred to as Constriction of Mitochondrial Inner Compartments (CoMIC). While this intra-mitochondrial event occurs at ERmitochondrial contact sites, it appears to be initiated independently of Drp1 action and OMM constriction. We provide evidence that CoMIC is initiated by an influx of $\mathrm{Ca}^{2+}$ into the mitochondria, which mediates subsequent mitochondrial influx of $\mathrm{K}^{+}$and Opal activation of the IMM. We propose that this regulatory mechanism can coordinate IMM morphological changes with OMM constriction for efficient mitochondrial division.

\section{Results}

Elongated mitochondria exhibit CoMIC before division. To explore the intra-mitochondrial events that take place during mitochondrial division, we performed time-lapse imaging in rat cultured cortical neurons expressing DsRed-mito, which labels the mitochondrial matrix ${ }^{26}$. Interestingly, a subset of long mitochondria in neuronal processes spontaneously exhibited repetitive and reversible beads-on-a-string-like structures accompanying focal constriction and bulging (Fig. 1a and Supplementary Movie 1). On the other hand, co-expressed green fluorescent protein (GFP) signals were not altered, ruling out the possibility that the observed mitochondrial change is influenced by morphological alteration of neuronal processes. This phenomenon was also observed in other cell types, including 293T, HeLa and HepG2 cells (Supplementary Fig. 1a), indicating that CoMIC is common to diverse cell types and species. Through kymographic analysis, we temporally and spatially analyzed the occurrence of CoMICs and mitochondrial division. The bulged and constricted sites were spatially conserved during repetitive CoMICs (Fig. 1b), and each mitochondrion exhibited unique and unsynchronized patterns of CoMIC spikes (Fig. 1c). These properties imply that CoMICs may be an intrinsic property of mitochondria. In young neurons (days in vitro 4, DIV4), the probability that a mitochondrion shows a CoMIC in a 10-min time period was $12.3 \%$, and in mature neurons (DIV10), which have more elongated mitochondria ${ }^{27}$, the probability was $35.6 \%$ (Fig. 1d). Notably, mitochondria longer than $5 \mu \mathrm{m}$ have a significantly higher probability of CoMIC (young neurons, $50 \%$; mature neurons, 61.3\%) than do shorter mitochondria (young neurons, 7.4\%; mature neurons, 10.5\%) (Fig. 1e). This indicates that elongated mitochondria are prone to CoMIC. In addition, mitochondria exhibiting CoMIC often underwent subsequent division at only one of multiple constricted sites (Fig. 1a-c). Further quantitative analyses revealed that 54.1 and $61.3 \%$ of mitochondria exhibiting CoMIC underwent subsequent division in young and mature neurons, respectively (Fig. 1e), and that larger proportions $(61.5 \%$ and $81.7 \%$, respectively) of dividing mitochondria exhibited prior CoMIC (Fig. 1f). These results suggest that CoMIC is closely correlated with mitochondrial division.

CoMIC is potentiated by suppression of Drp1. We further examined the association of CoMIC with mitochondrial division by overexpression of a GTPase-defective mutant of Drp1 (DNDrp1), which inhibits the activity of endogenous Drp1 (ref. 28), resulting in marked elongation of mitochondria with a reduced rate of division (Supplementary Fig. 2). By using other chemical and genetic dyes, we confirmed that the CoMIC was real but not 
a
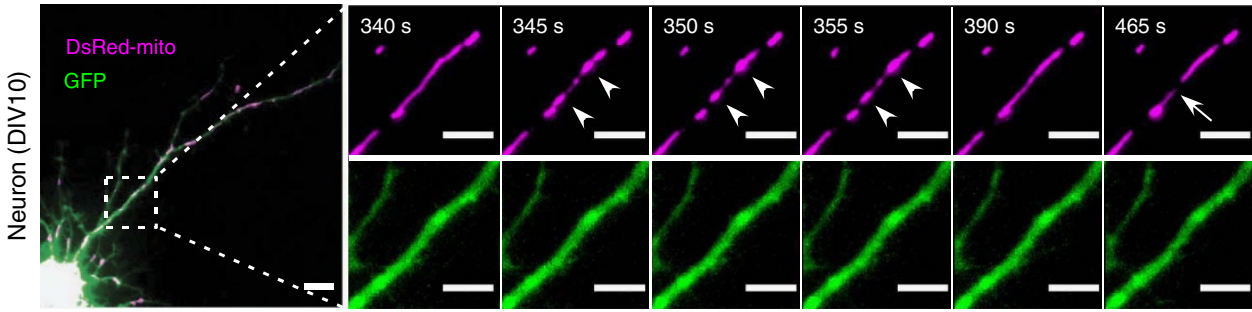

b

C
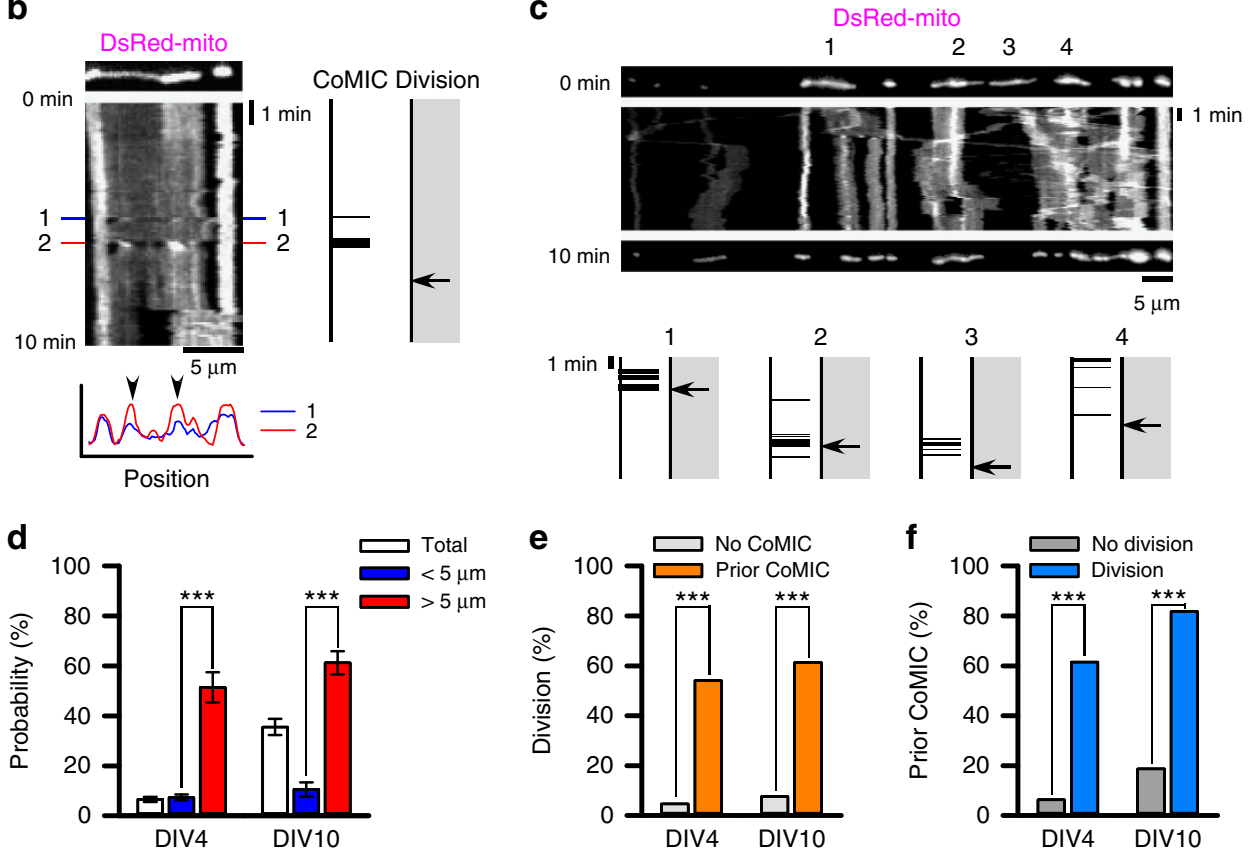

Figure 1 | CoMIC appears in elongated mitochondria before mitochondrial division. (a) Time-lapse images of mitochondrial matrices (DsRed-mito) in neuronal process (GFP). The white arrowheads and the arrow indicate sites of mitochondrial bulging and division, respectively. Scale bars, 10 and $5 \mu \mathrm{m}$ in the lower and higher magnification images, respectively. (b) Kymograph of DsRed-mito in $\mathbf{a}$. The lower graph is the intensity profile at two CoMIC-showing time points $(1,2)$, and arrow heads indicate conserved bulging site. Two illustrations on the right indicate the pattern of CoMIC and occurrence of division. CoMIC pattern and division are labelled by spikes and an arrow, respectively. (c) Kymograph of DsRed-mito in a neuronal process. The upper numbers indicate mitochondria showing CoMIC. The lower panels show the pattern of CoMIC and occurrence of division in mitochondria labelled with the numbers above. (d) Probability of CoMIC in mitochondria from young (DIV4; $<5 \mu \mathrm{m}=530$ mitochondria, $>5 \mu \mathrm{m}=70$ mitochondria) and mature neurons (DIV10; $<5 \mu \mathrm{m}=114$ mitochondria, $>5 \mu \mathrm{m}=111$ mitochondria) during a 10-min time period ( ${ }^{\star \star \star} P<0.01$ by the Mann-Whitney rank sum test). Error bars indicate s.e. (e) Proportion of mitochondria undergoing subsequent division among mitochondria showing no or prior CoMIC ( ${ }^{\star \star \star} P<0.01$ by the $\chi^{2}$-test with Yates's

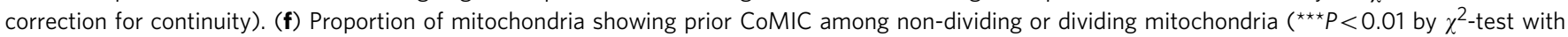
Yates's correction for continuity).

DsRed-specific artifact (Supplementary Fig. 3). Strikingly, DNDrp1-expressing neurons exhibited potentiated frequency and duration of CoMIC compared with control neurons (Fig. 2a and Supplementary Movie 2), although the probability of CoMIC was not affected when compared with elongated mitochondria (Fig. 2b). This phenomenon was also found in other cell types, including 293T, HeLa and HepG2 cells (Supplementary Fig. 1b), implying that the CoMIC-enhancing effect of DN-Drp1 is conserved in cells of diverse types and species. Kymographic analysis revealed that CoMIC was more frequent and prolonged in the mutant compared with control neurons (Fig. 2c). Further quantitative analyses showed significant increases in total duration, frequency and mean interval of CoMIC in DN-Drp1-expressing neurons compared with control neurons (Fig. 2d). In addition, mitochondrial bulging during CoMIC was also enhanced by DNDrp1 (Fig. 2e,f). These results show that Drp1 relieves mitochondria from the tension caused by CoMIC by executing mitochondrial division. It is known that DN-Drp1 maintains strong oligomerization activity and affinity for the lipid membrane $e^{29}$. In addition, photo-damage or hypoxic stress may induce CoMIC-like morphology resulting from inhibition of Drp1 on the $\mathrm{OMM}^{22,25}$. Therefore, we wondered if the overexpression of DN-Drp1 promoted CoMIC via enhanced mitochondrial constriction with perturbation of division. However, knockdown of endogenous Drp1 with a small hairpin RNA (shRNA) similarly promoted CoMIC (Supplementary Fig. 4). Crucially, Drp1-knockout mouse embryonic fibroblasts also exhibited CoMIC (Fig. 2g). These data suggest that Drp1 is not required for the initiation of CoMIC, but may terminate CoMIC cycles by mitochondrial division.

ER-mitochondria contact site spatially specifies CoMIC spots. Recent studies revealed that physical association of ERmitochondria and actin filaments can trigger mitochondrial constriction before recruitment of Drp1 (refs 7,8). Our kymographic analyses showed that mitochondrial constriction occurs in spatially conserved sites throughout repetitive CoMIC 

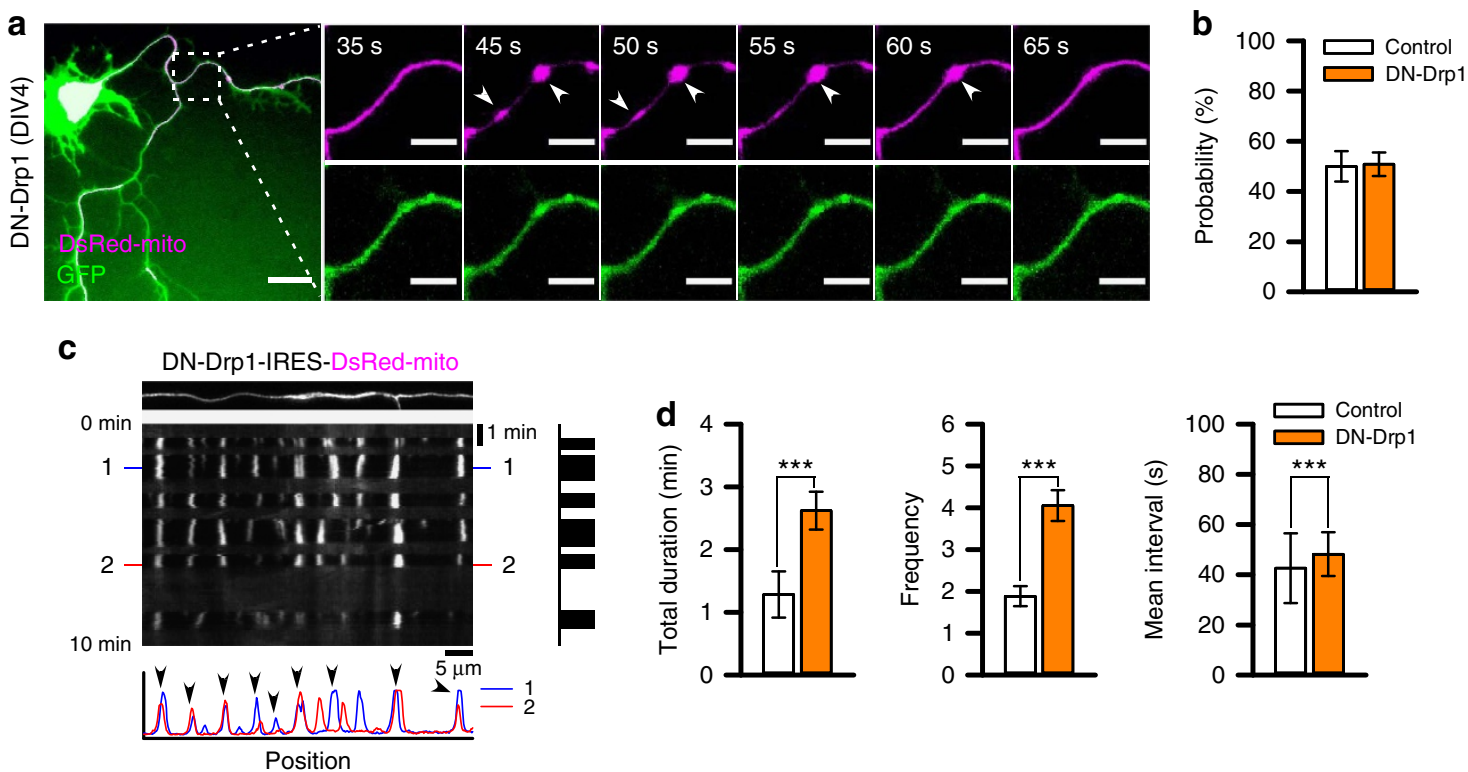

e

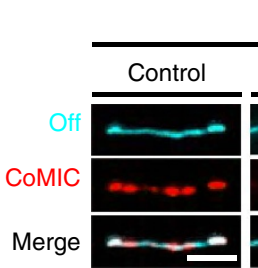

DsRed-mito
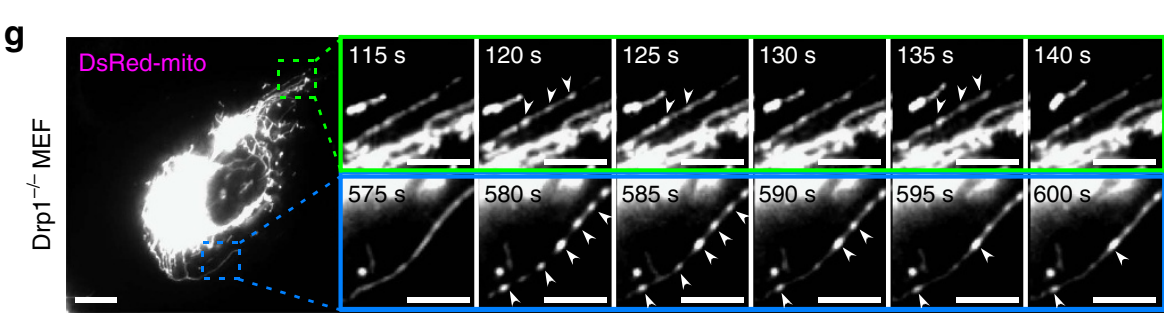

Figure 2 | CoMIC is potentiated by inhibition of Drp1, but does not depend on Drp1. (a) Time-lapse images of the mitochondrial matrix and neuronal processes in DN-Drp1-expressing neurons (DIV4). Arrowheads indicate sites of mitochondrial bulging. Scale bars, 10 and $5 \mu \mathrm{m}$ in the lower and higher magnification images, respectively. (b) Probability of CoMIC in elongated mitochondria ( $>5 \mu \mathrm{m}$ ) as compared with control (70 mitochondria) and DNDrp1-expressing neurons (114 mitochondria). Error bar indicate s.e. (c) Representative kymographic analysis of a mitochondrion showing CoMIC in a DNDrp1-expressing neuron. Arrow heads indicate conserved bulging site on two CoMIC-showing time points (1, 2). (d) Quantification of the total duration, frequency and mean interval of CoMIC in control and DN-Drp1-expressing neurons ( ${ }^{\star \star \star} P<0.01$ by Mann-Whitney rank sum test). (e) Straightened images of DsRed-mito showing minimal bulging (cyan signal) and maximal bulging (red signal) in control and DN-Drp1-expressing neurons. (f) Bulging ratio in foci during CoMIC in control and DN-Drp1-expressing neurons ( ${ }^{\star \star \star} P<0.01$ by Mann-Whitney rank sum test). (g) CoMIC in Drp1 knockout mouse embryonic fibroblasts. The time-lapse panels are enlarged in the green and blue boxes. Arrowheads indicate the sites of mitochondrial bulging. Scale bars, 10 and $5 \mu \mathrm{m}$ in the lower and higher magnification images, respectively.

(Figs le and $2 \mathrm{~b}$ ), implying the presence of structural foci for CoMIC. We thus postulated that spatial association with the ER may specify the position at which CoMIC occurs. As expected, CoMIC and subsequent mitochondrial division occurred when mitochondria spatially intersected with or were enclosed by the ER, as assessed by labelling with GFP-Sec61b (Fig. 3a,b and Supplementary Fig. 5a). DN-Drp1-expressing neurons and 293T cells also exhibited CoMIC at ER-mitochondrial intersections (Fig. 3c,d, Supplementary Movie 3 and Supplementary Fig. 5b-d). In addition, GFP-Mff, which marks putative sites of constriction and division in ER-mitochondria contact site $^{7}$, localized on constriction sites during CoMIC in control and DN-Drp1expressing neurons (Fig. 3e,f). More recently, it has been demonstrated that replication of mitochondrial DNA (mtDNA) is spatially associated with mitochondrial division in ER-mitochondria contact site ${ }^{30}$. By using GFP-tagged Twinkle which is mtDNA helicase for replication ${ }^{31,32}$, we examined the positions where mtDNA replisomes localize during CoMIC. In off-phase, Twinkle-GFP signals were localized on the elongated mitochondria, and mitochondrial constrictions occurred juxtaposition of Twinkle-GFP during CoMIC (Fig. 3g,h). Therefore, we suggest that structural specification of CoMIC is associated with ER-mitochondrial contact site. Remarkably, however, during CoMIC, the spatial intersections between the ER and mitochondria seems to be maintained regardless of phase, and some intersections did not show any CoMIC (Fig. 3a-d and Supplementary Fig. 5b). However, depletion of Mfn2, which regulates ER-mitochondria contact ${ }^{33,34}$, did not modify DN- 
a

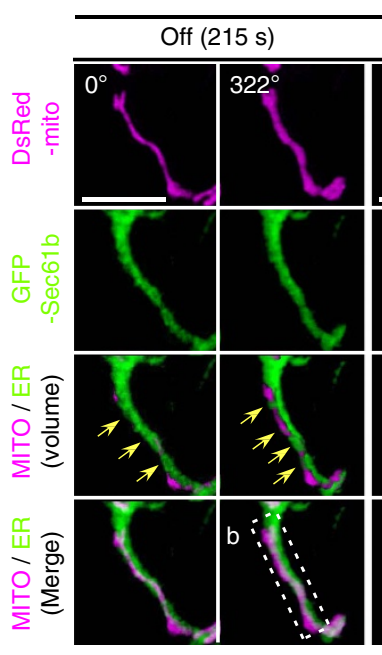

b

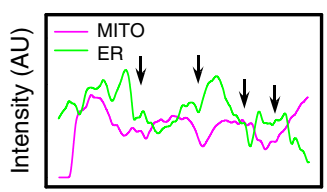

Control
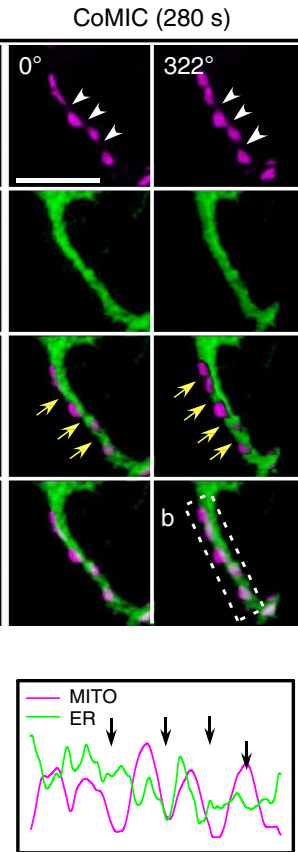

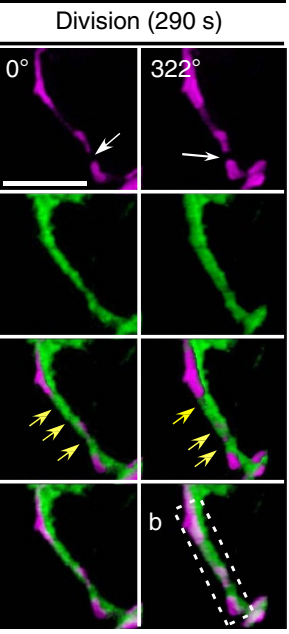

C

\begin{tabular}{|c|c|}
\hline \multicolumn{2}{|c|}{ DN-Drp1 } \\
\hline CoMICs (30 s) & Off $(120 \mathrm{~s})$ \\
\hline
\end{tabular}
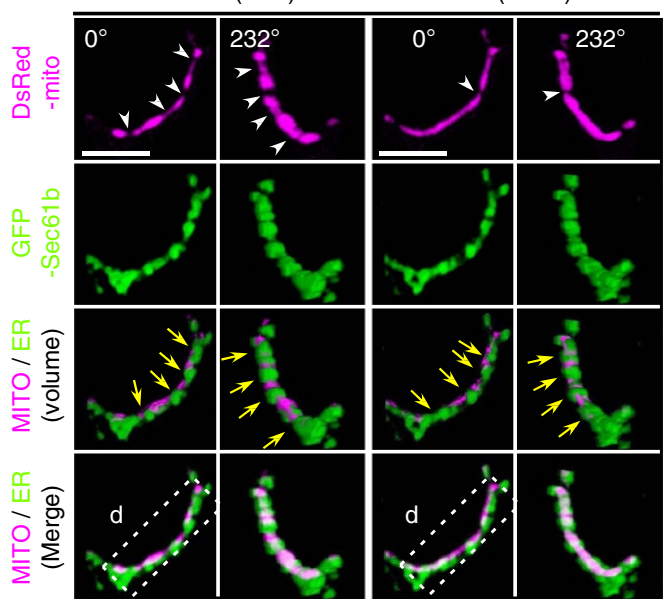

d
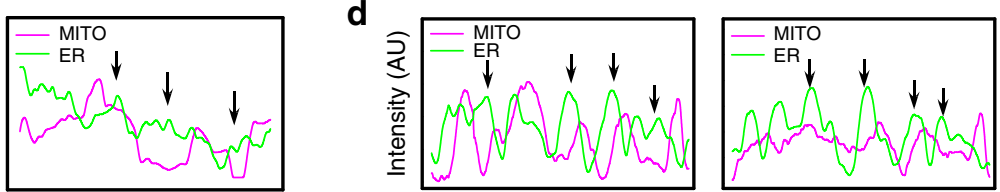

e
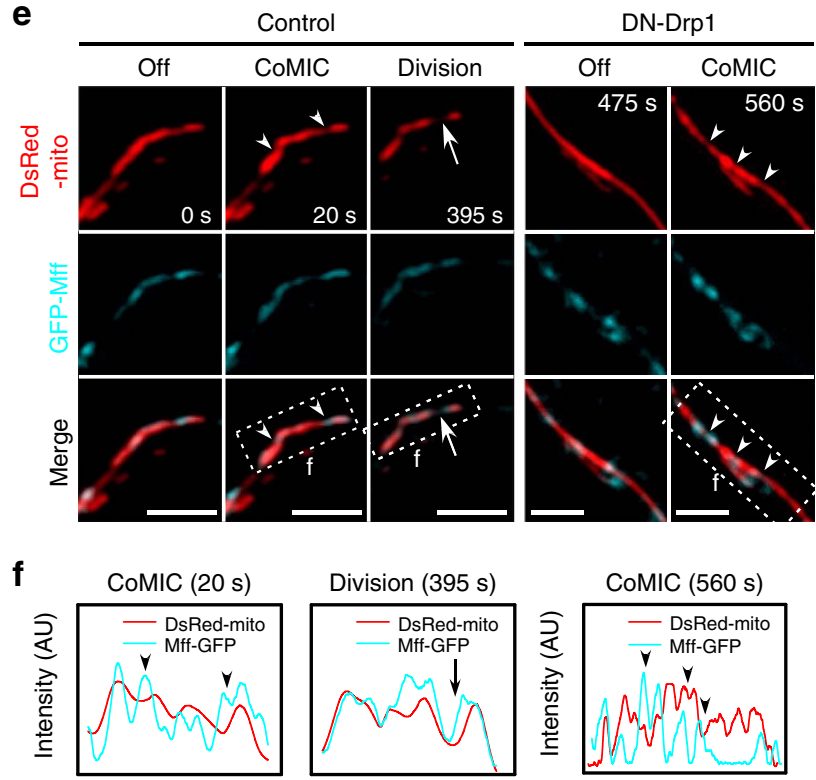

g
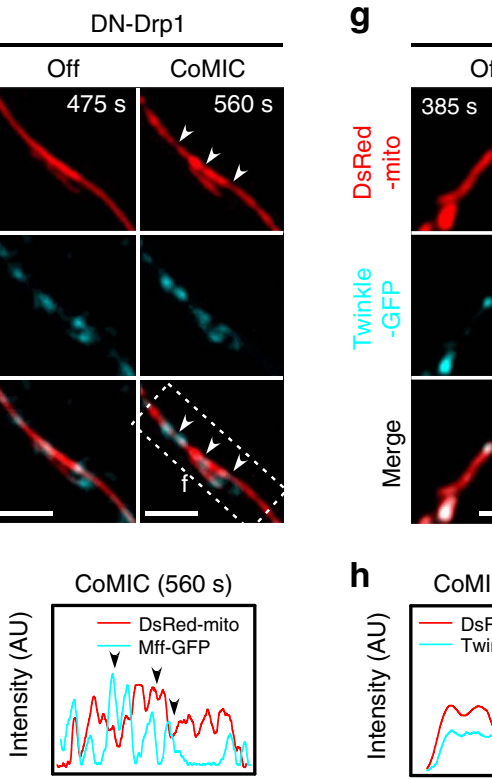

Control
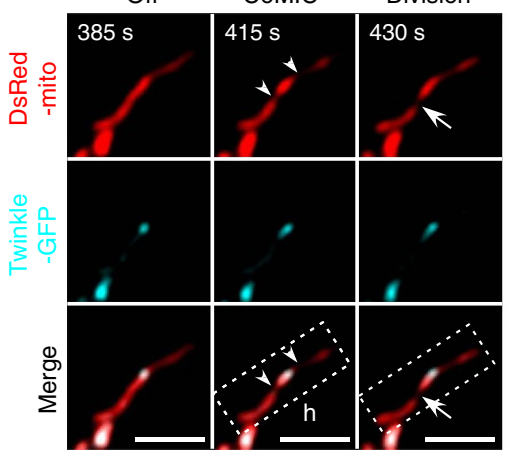

h $\quad$ CoMIC $(415 \mathrm{~s}) \quad$ Division $(430 \mathrm{~s})$
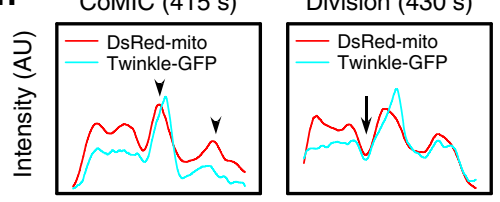
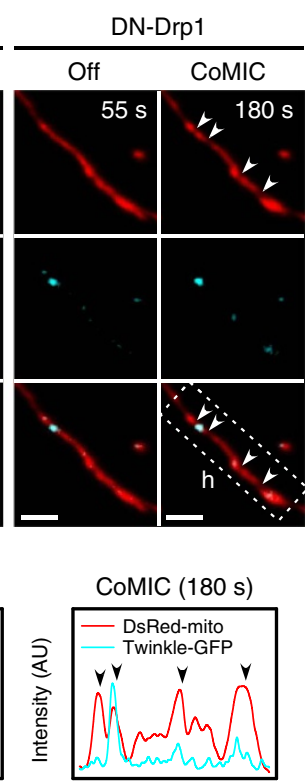

i
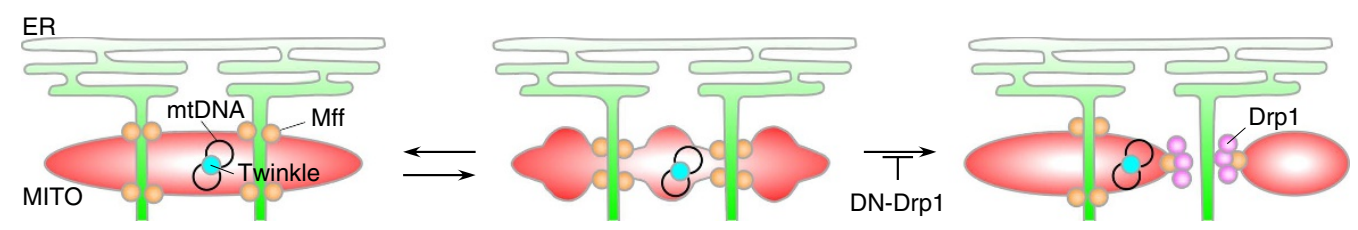

Figure 3 | CoMIC is spatially associated with the ER. (a,c) 3D images of mitochondria and ER (GFP-Sec61b) during off-phase, CoMIC and mitochondrial division in control (a) and DN-Drp1-expressing neurons (c). Scale bar, $5 \mu \mathrm{m}$. White arrowheads and arrows indicate the spots of CoMIC and division, respectively. Yellow arrows indicate the spots that are enclosed by the ER. (b,d) Intensity profiles of the mitochondrial matrix and the ER in the dotted boxes in a,c, respectively. (e,g) Representative images of mitochondria, GFP-Mff (e), Twinkle-GFP (g) during off-phase, CoMIC and mitochondrial division in control and DN-Drp1-expressing neurons. Scale bar, $5 \mu \mathrm{m}$, and white arrowheads and arrows indicate the spots of CoMIC and division, respectively. $(\mathbf{f}, \mathbf{h})$ Intensity profiles of the mitochondrial matrix, GFP-Mff and Twinkle-GFP in the dotted boxes in e,g, respectively. (i) A hypothetical model of mitochondrial association with the ER during COMIC. 
Drp1-potentiated CoMIC (Supplementary Fig. 6), similar to previous report ${ }^{7}$. These data indicate that contact with the ER per se does not induce CoMIC but most likely plays a role in the structural specification of CoMIC (Fig. 3i). We further examined whether actin filaments are involved in induction of CoMIC because actin polymerization triggers constriction of the OMM before Drp1-dependent division ${ }^{8}$. However, treatment with latrunculin B, which disrupts actin filaments, did not modify CoMIC (Supplementary Fig. 7). Collectively, these data suggest that the cytosolic vesicle-forming machinery, such as actin filaments and Drp1, are not involved in the induction of CoMIC, while ER-mitochondrial intersections spatially specify the foci of CoMIC.

CoMIC is independent on OMM changes. Because cytosolic fission machinery is not involved in CoMIC, we wondered the behaviour of the OMM during CoMIC. Interestingly, a subset of OMM, which was labelled with Tom20-GFP, in elongated mitochondria exhibited neither constriction nor bulging during CoMIC in control neurons, but underwent subsequent division (Fig. 4a,b nd Supplementary Fig. 8a). Such dissociation of CoMIC and OMM changes was also observed in 293T cells (Supplementary Fig. 8b). In some cases, CoMIC and OMM constrictions were simultaneously observed, along with conservation of bulging spots. Similarly, neurons and 293T cells expressing DN-Drp1 exhibited no or marginal OMM bulging during potentiated CoMIC (Fig. 4c,d, Supplementary Movie 4 and Supplementary Fig. 8c). The quantification confirmed that the probability of focal OMM constriction was much lower than that of CoMIC (Fig. 4e). In addition, bulging of the OMM is weaker than that of CoMIC (Fig. 4f,g), implying the dissociation of OMM and IMM at constriction sites during CoMIC. Supporting this, electron microscopy in shDrpl-infected neuron showed that some elongated mitochondria exhibit focal separation of IMM and OMM (Fig. 4h). To visualize the distance of OMM-IMM during CoMIC, we performed proximity assay by fluorescence resonance energy transfer (FRET) between mitochondria matrix and OMM, labelled by mito-BFP and Tom20GFP, respectively (Supplementary Fig. 9). We expected that proximity between IMM and OMM may be represented by FRET signal, and found some hot and cold spots were shown on mitochondria (Fig. 4i). Strikingly, among the hot spots, bulging of mitochondrial matrix occurred at some spots with enhanced proximity between OMM and IMM during CoMIC compared with off-phase (Fig. 4i,j). After CoMIC, these hot spots returned to their basal level. On the other hand, the cold spots became striking at the constriction sites during CoMIC (Fig. 4i,j), indicating that constriction of mitochondrial matrix increases OMMIMM distances. Notably, the hot and cold spots appeared to be spatiotemporally conserved with moderate dynamicity during offphase (Fig. 4k). Therefore, these data suggest that CoMIC induces transient disorganization of OMM-IMM contact.

Mitochondrial $\mathrm{Ca}^{2+}$ initiates and potentiates CoMIC. Spatial association of CoMIC with ER may indicate the $\mathrm{Ca}^{2+}$-mediated functional linkage between CoMIC and ER. Especially, intra-mitochondrial $\mathrm{Ca}^{2+}$ entry is implicated in many mitochondrial functions, including respiration, apoptosis, reactive oxygen species production and mitochondrial transport ${ }^{35,36}$. To investigate involvement of intra-mitochondrial $\mathrm{Ca}^{2+}$ in CoMIC, we monitored the level of intra-mitochondrial $\mathrm{Ca}^{2}+$ during CoMIC by using CEPIA3mt (ref. 37). Notably, intra-mitochondrial $\mathrm{Ca}^{2+}$ was transiently increased just before the CoMIC in DN-Drp1expressing neuron, and returned to basal level in off-phase (Fig. 5ac) mitochondrion \#2). However, some mitochondria did not undergo CoMIC in spite of mitochondrial $\mathrm{Ca}^{2}+$ rise (Fig. 5a-c, mitochondrion \#1). It implies that mitochondrial $\mathrm{Ca}^{2+}$ signal may be necessary, but not sufficient, for CoMIC. Next, we examined the role of $\mathrm{Ca}^{2+}$ in CoMIC. Treatment with BAPTA$\mathrm{AM}$, a cell-permeable $\mathrm{Ca}^{2}+$ chelator, completely inhibited CoMIC in DN-Drp1-expressing neurons (Supplementary Fig. 10a,b). However, ethylene glycol-bis( $\beta$-aminoethyl ether)-N,N, $\mathrm{N}^{\prime}, \mathrm{N}^{\prime}$ tetraacetic acid (EGTA), a non-cell-permeable $\mathrm{Ca}^{2}+$ chelator, did not affect CoMIC (Supplementary Fig. 10c). In contrast, A23187, which is a $\mathrm{Ca}^{2+}$ ionophore, induced robust CoMIC and eventual mitochondrial fragmentation (Supplementary Fig. 10d). These data indicate that intracellular $\mathrm{Ca}^{2}+$ rise can induce CoMIC. In consistent, 2-APB, which is an inhibitor of $\mathrm{IP}_{3}$ receptor releasing $\mathrm{Ca}^{2}+$ from ER, significantly declined the probability of basal CoMIC and shortened its total and average single durations (Fig. 5d,e), implying the contribution of intra-mitochondrial $\mathrm{Ca}^{2+}$ shuttling from ER to CoMIC. To more selectively explore the involvement of intra-mitochondrial $\mathrm{Ca}^{2}+$ entry in CoMIC initiation, we treated DN-Drp1-expressing neurons with Ru360, an inhibitor of mitochondrial $\mathrm{Ca}^{2+}$ uptake. Remarkably, treatment with Ru360 inhibited CoMIC as compared with vehicle (DMSO) treatment (Fig. 5f,g). Quantitative analyses revealed that Ru360 significantly reduced the probability of CoMIC, and shortened the average duration of an individual CoMIC (Fig. 5j). Furthermore, Ru360 efficiently blocked CoMIC induced by thapsigargin (TG), which increases intracellular $\mathrm{Ca}^{2}+$ concentration by blocking calcium uptake via inhibition of sarco/ER $\mathrm{Ca}^{2+}$ ATPase (Fig. 5hj). Similarly, depletion of $\mathrm{Mcu}$ by specific shRNA, which is mitochondrial uniporter, decreased the probability of CoMIC and shortened total duration of CoMIC (Fig. 5k,l), although it did not affect mitochondrial morphology (Supplementary Fig. 11). Taken together, these data suggest that intra-mitochondrial $\mathrm{Ca}^{2+}$ entry is required for the induction and potentiation of CoMIC.

Mitochondrial $\mathrm{Ca}^{2+}$ promotes efficient mitochondrial division. Previous reports have shown that intra-mitochondrial $\mathrm{Ca}^{2}+$ entry is involved in mitochondrial division, although precise molecular mechanism was yet unclear ${ }^{38-41}$. Since CoMIC occurs before mitochondrial division and is mediated by intra-mitochondrial $\mathrm{Ca}^{2+}$, we tested the $\mathrm{Ca}^{2+}$-mediated functional link between CoMIC and mitochondrial division. First, we monitored the level of intra-mitochondrial $\mathrm{Ca}^{2+}$ during CoMIC and subsequent mitochondrial division in control neurons. Similarly to DN-Drp1expressing neurons, control neuron also exhibited spontaneous, transient and dramatic increment of intra-mitochondrial $\mathrm{Ca}^{2+}$ just before and during CoMIC, and it returned to basal level during off-phase (Fig. 6a-c). However, there was no remarkable fluctuation of intra-mitochondrial $\mathrm{Ca}^{2+}$ during mitochondrial division. These data indicate that intra-mitochondrial $\mathrm{Ca}^{2+}$ is involved in induction of CoMIC but not Drp1-mediated mitochondrial division. Next, we examined the effect of intramitochondrial $\mathrm{Ca}^{2+}$ on mitochondrial length. Treatment with Ru360 significantly increased mitochondrial length and attenuated TG-induced mitochondrial fragmentation (Fig. 6d,e). Consistently, depletion of $\mathrm{Mcu}$ also significantly decreased mitochondrial length (Fig. 6f,g). These data indicate that intra-mitochondrial $\mathrm{Ca}^{2+}$ entry promotes mitochondrial division as well as CoMIC. Considering that mitochondrial division does not always accompany with fluctuation of intra-mitochondrial $\mathrm{Ca}^{2+}$, we suggest that intra-mitochondrial $\mathrm{Ca}^{2}+$ indirectly promotes mitochondrial division by promoting CoMIC.

Mitochondrial bulging and depolarization is mediated by $\mathbf{K}^{+}$. Mitochondrial bulging or swelling is induced by osmotic movement of water molecules into the mitochondrial matrix, and this 

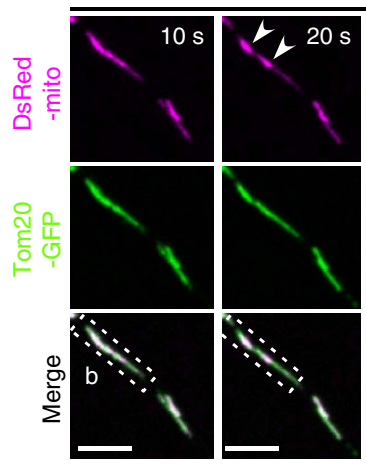

Contro
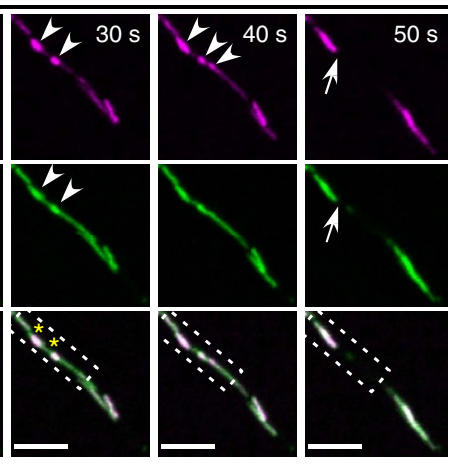

b

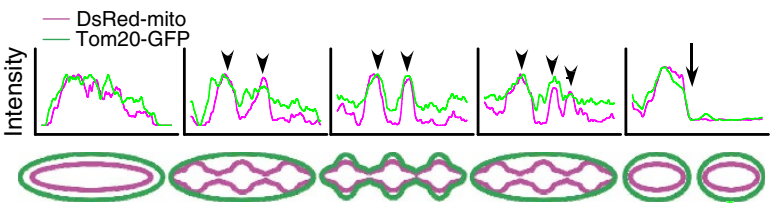

e

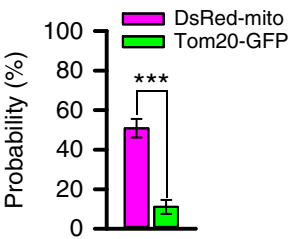

f

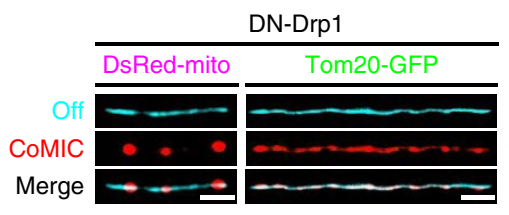

i

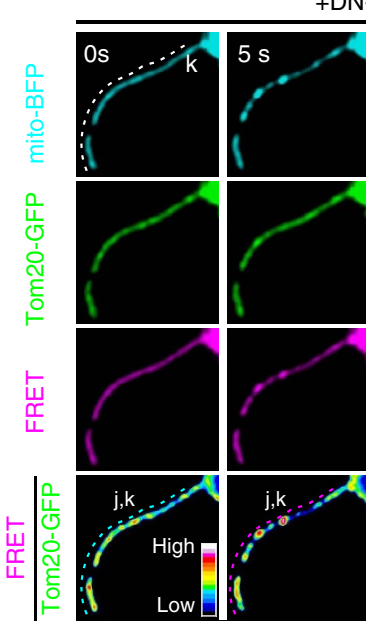

+DN-Drp1
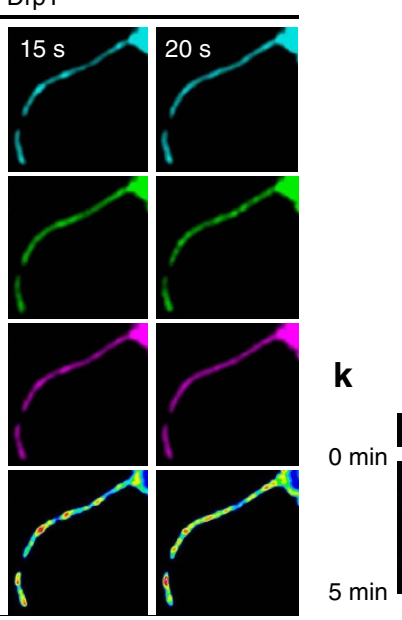

k
C
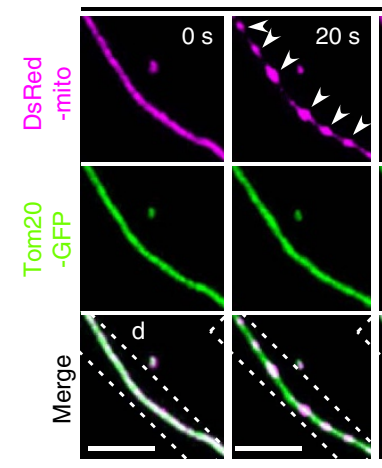

+ DN-Drp1

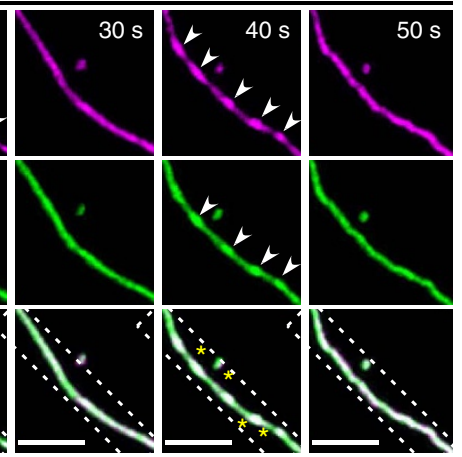

d $\quad$ DsRed-mito

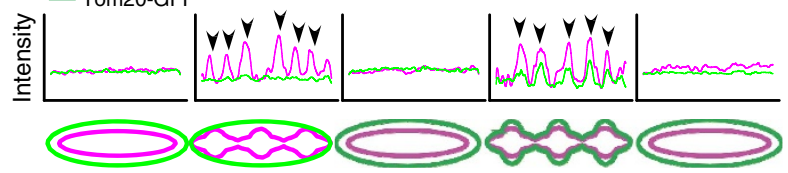

g

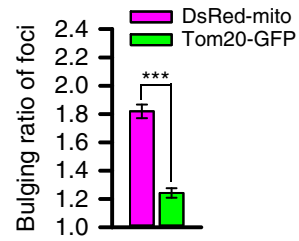

h

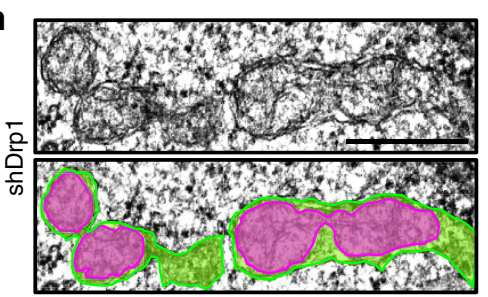

j

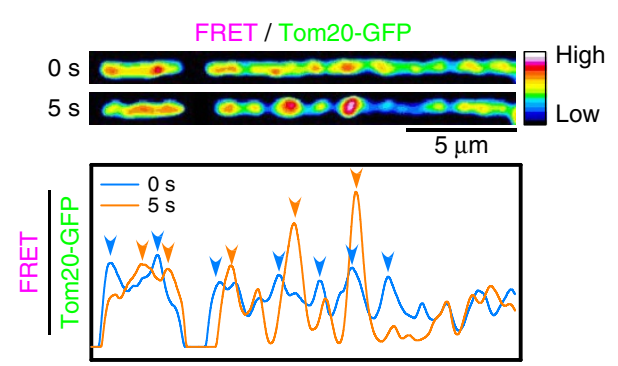

mito-BFP

FRET / Tom20-GFP

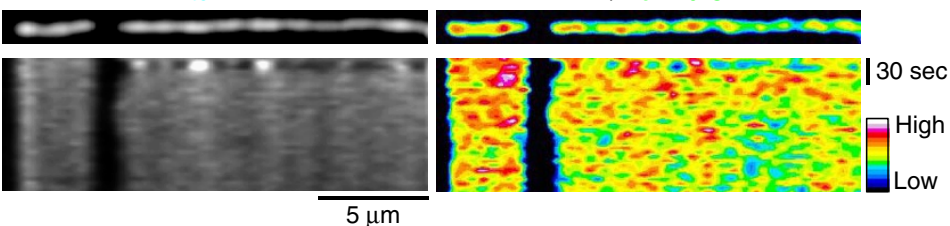

Figure 4 | CoMIC is OMM-independent event accompanying rearrangement on IMM-OMM contact. (a,c) Time-lapse images of the mitochondrial matrix and OMM (Tom20-GFP) in control (a) and DN-Drp1-expressing neurons (c). Arrowheads and arrows indicate the bulging sites of mitochondria during CoMIC and mitochondrial division, respectively. Yellow asterisks indicate synchronized bulging of the OMM and matrix. Scale bar, $5 \mu \mathrm{m}$. (b,d) Intensity profiles (upper graphs) and schematic diagrams (lower graphs) of the mitochondrial matrix (magenta lines) and OMM (green lines) in the dotted insects shown in a,c, respectively. (e) Probability of CoMIC (114 mitochondria) and OMM constriction (81 mitochondria) in elongated mitochondria from DN-Drp1-expressing neurons ( ${ }^{\star \star \star} P<0.01$ by Mann-Whitney rank sum test). Error bar indicate s.e. (f) Straightened images of DsRed-mito and Tom20-GFP showing minimal (cyan signal) and maximal bulging (red signal) in a DN-Drp1-expressing neuron. (g) Bulging ratio in foci of DsRed-mito and Tom20-GFP during CoMIC in DN-Drp1-expressing neurons ( ${ }^{\star \star \star} P<0.01$ by Mann-Whitney rank sum test). (h) Electron microscopy of mitochondria in neuron infected by shDrp1-virus. Scale bar, $500 \mathrm{~nm}$. Green and magenta shades indicate area marked by OMM and IMM, respectively. (i) Time-lapse images for FRETbased proximity analysis between OMM and mitochondrial matrix, which are labelled by Tom20-GFP and mito-BFP, in a DN-Drp1-expressing neuron. Scale bar, $5 \mu \mathrm{m}$. FRET ratio (FRET/Tom20-GFP) is pseudo-coloured. (j) Straightened images of FRET/Tom20-GFP during off-phase and CoMIC of mitochondria, and their intensity profiles (lower panel). Arrows indicate hot spots of FRET ratio. (k) Kymographies of mito-BFP and FRET/Tom20-GFP of mitochondria on dotted line of $\mathbf{i}$. 
a

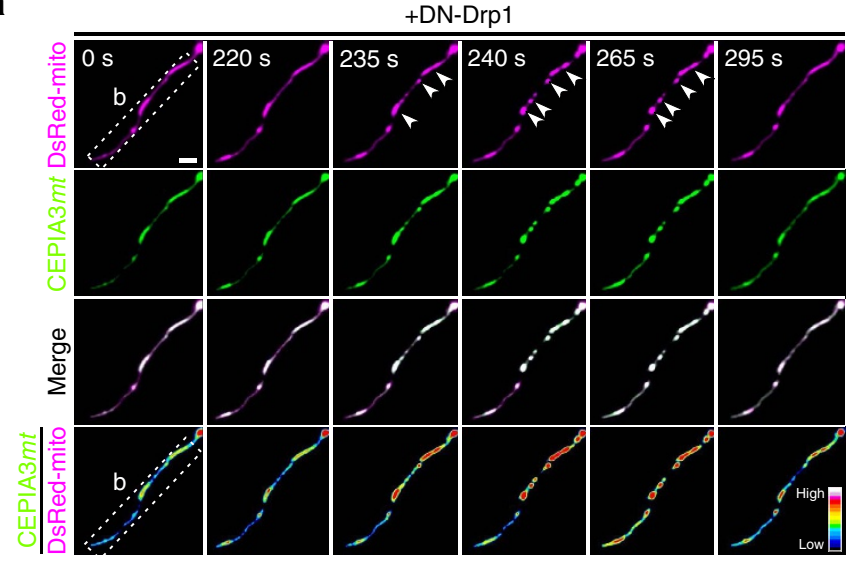

b

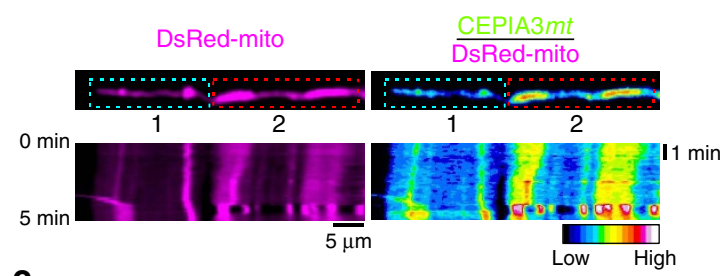

C

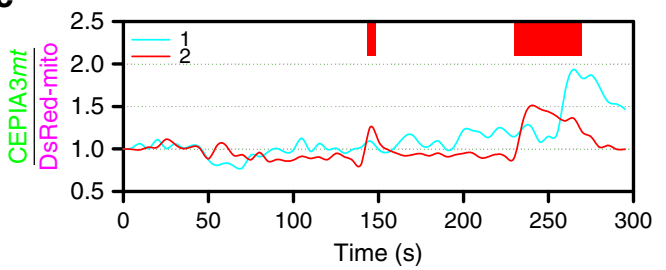

d

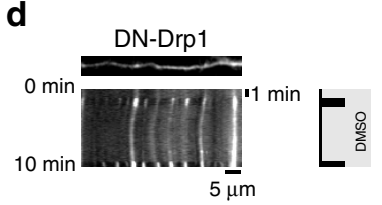

$\mathbf{f}$
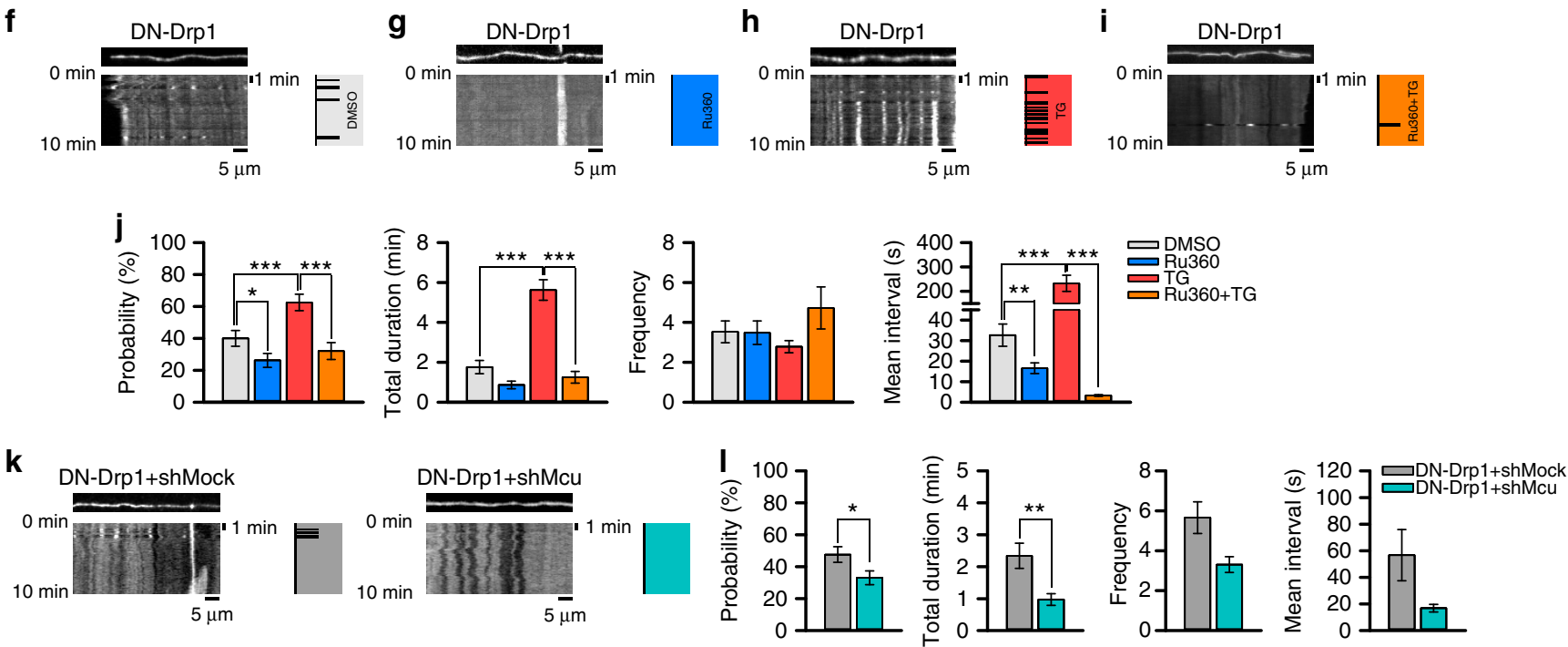

Figure 5 | Intra-mitochondrial $\mathbf{C a}^{\mathbf{2}}+$ regulates CoMIC. (a) Time-lapse images of DsRed-mito and CEPIA3mt in DN-Drp1-expressing neuron. Arrow heads indicate mitochondrial bulging. Scale bar, $5 \mu \mathrm{m}$, and CEPIA3mt/DsRed-mito is pseudo-coloured. (b) Kymographies of DsRed-mito and CEPIA3mt/DsRedmito of mitochondria in dotted box of $\mathbf{a}$. (c) Temporal CEPIA3mt/DsRed-mito fluctuation of mitochondria of dotted boxes (\#1, \#2) of $\mathbf{b}$. Upper red boxes indicate CoMIC duration of mitochondrion labelled by \#2. (d) Representative kymographies of mito-GFP and patterns of CoMIC (right diagram) in DNDrp1-expressing neurons treated with DMSO and 2-APB $(1 \mu \mathrm{M})$ for $1 \mathrm{~h}$. (e) Probability of CoMIC in elongated mitochondria treated with DMSO (103

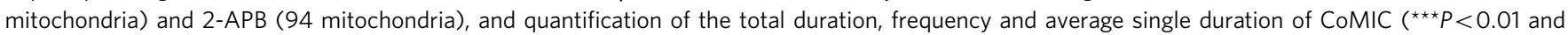
${ }^{\star} P<0.05$ by Mann-Whitney rank sum test). Error bar indicate s.e. (f-i) Representative kymographies of DsRed-mito and patterns of CoMIC (right diagram) in DN-Drp1-expressing neurons treated with DMSO (f), Ru360 (10 $\mu \mathrm{M})(\mathbf{g})$, thapsigargin (TG, $1 \mu \mathrm{M})(\mathbf{h})$ and TG with 30 min pretreatment with Ru360 (Ru360 + TG) (i) for $1 \mathrm{~h}$. (j) Probability of CoMIC in elongated mitochondria treated with DMSO (100 mitochondria), Ru360 (95 mitochondria), TG (88 mitochondria) or Ru360 + TG (95 mitochondria), and quantification of the total duration, frequency and average single duration of CoMIC ( ${ }^{\star \star \star} P<0.01$, ${ }^{*} P P<0.02$ and ${ }^{\star} P<0.05$ by Mann-Whitney rank sum test). (k) Representative kymographies of DsRed-mito and patterns of CoMIC (right diagram) in DNDrp1 + shMock- and DN-Drp1 + shMcu-expressing neurons. (I) Probability of CoMIC in elongated mitochondria from DN-Drp1+shMock- (105 mitochondria) and DN-Drp1 + shMcu-expressing neurons (118 mitochondria), and quantification of the total duration, frequency and average single duration of CoMIC $\left({ }^{\star \star} P<0.02\right.$ and ${ }^{\star} P<0.05$ by Mann-Whitney rank sum test).

process is mainly regulated by $\mathrm{K}^{+}$influx controlled by the mitochondrial big-conductance $\mathrm{Ca}^{2+}$-dependent $\mathrm{K}^{+}$channel ( mitoBK $_{\mathrm{Ca}}$ ) and ATP-dependent $\mathrm{K}^{+}$channel (mitoK $\mathrm{ATP}$ ), which finally leads to an increase in osmotic pressure between the cytoplasm and the mitochondrial matrix ${ }^{19}$. Considering that intra-mitochondrial $\mathrm{Ca}^{2}+$ induces CoMIC accompanied by mitochondrial bulging, we investigated the possible involvement of mitoBK $\mathrm{Ca}$ in CoMIC. Pre-treatment with paxilline (Pax), an inhibitor of mitoBK $\mathrm{Ca}_{\mathrm{Ca}}$, suppressed the probability of TG-induced, but not basal, CoMIC (Fig. 7a-c), but it did not affect the total duration, frequency or mean interval of CoMIC (Supplementary Fig. 12), implying partial involvement of induction/potentiation 

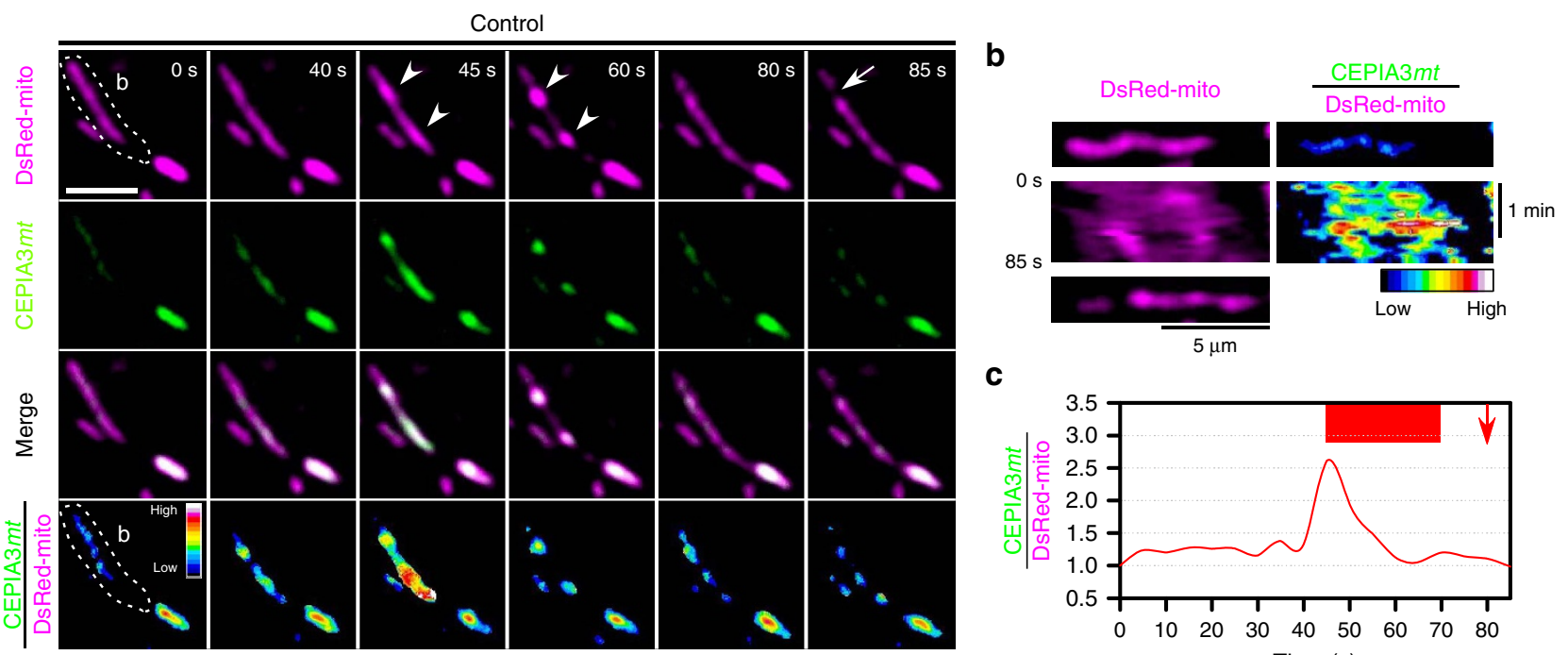

C

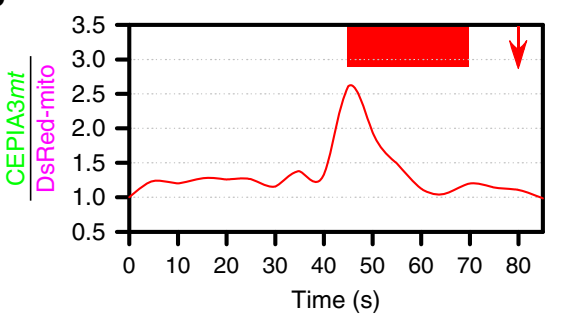

d

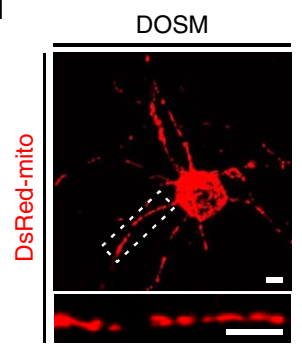

Ru360

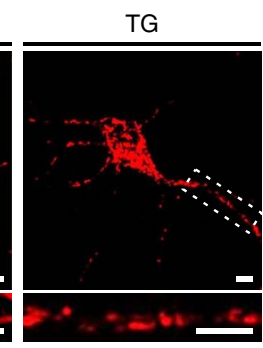

Ru360+TG
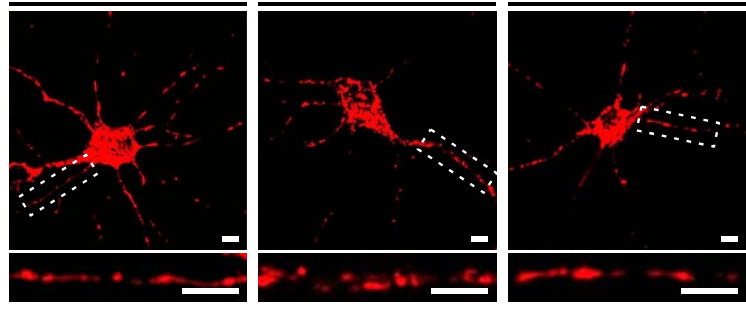

e

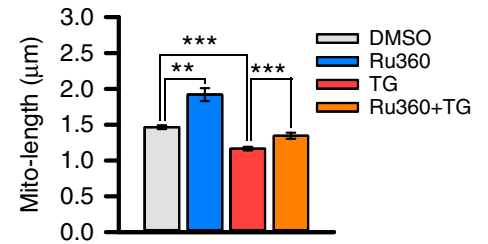

f
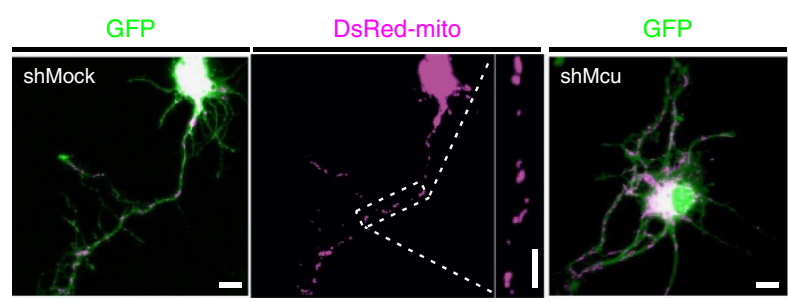

DsRed-mito

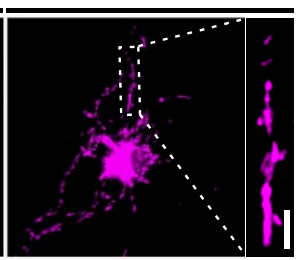

g

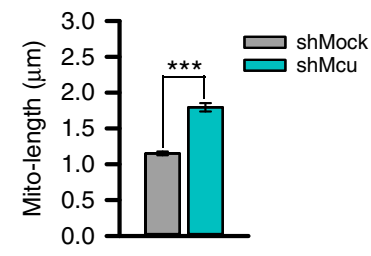

Figure 6 | Intra-mitochondrial $\mathbf{C a}^{2}+$ contributes to efficient mitochondrial division. (a) Time-lapse images of DsRed-mito and CEPIA3mt in control neuron. Scale bar, $5 \mu \mathrm{m}$, and CEPIA3mt/DsRed-mito is pseudo-coloured. Arrow heads and arrow indicate mitochondrial bulging and division, respectively. (b) Kymographies of DsRed-mito and CEPIA3mt/DsRed-mito of mitochondria in dotted region of a. (c) Temporal CEPIA3mt/DsRed-mito fluctuation of a mitochondrion of dotted region of $\mathbf{a}$. Upper red box and arrow indicate CoMIC duration and mitochondrial division, respectively. (d) Representative morphology of mitochondria in neurons treated with DMSO and Ru360 with or without TG. The lower, enlarged panels are straightened images from the boxes in the upper panels. Scale bar, $5 \mu \mathrm{m}$. (e) Average mitochondrial length following treatment with DMSO (2,415 mitochondria), Ru360 (635 mitochondria), TG (1,438 mitochondria) and TG + Ru360 (706 mitochondria). ( ${ }^{\star \star \star} P<0.01$ and ${ }^{\star \star} P<0.02$ by Mann-Whitney rank sum test). Error bar indicate s.e. (f) Representative morphology of mitochondria in shMock- and shMcu-expressing neurons. The enlarged right panels are straightened images from the dotted boxes. Scale bar, $5 \mu \mathrm{m}$. (g) Average mitochondrial length following treatment with shMock- (1,034 mitochondria) and shMcu-expressing neurons $\left(1,130\right.$ mitochondria). ( ${ }^{\star \star *} P<0.01$ by Mann-Whitney rank sum test).

of CoMIC unlike $\mathrm{Ca}^{2}+$. Instead, Pax efficiently prevented basal and TG-induced mitochondrial bulging during CoMIC (Fig. 6d,e). Previous studies have demonstrated that intramitochondrial influx of $\mathrm{K}^{+}$induces depolarization of mitochondria as well as bulging ${ }^{42,43}$. Recently, it has been also demonstrated that CoMIC accompanies with mitochondrial depolarization ${ }^{23,24}$. Consistently with these studies, we also found that mitochondria are transiently depolarized during CoMIC (Fig. 6f,g). Remarkably, Pax efficiently alleviated mitochondrial depolarization during CoMIC (Fig. 6f,h). On the other hand, prolonged treatment of NS1619, an opener of mitoBK $_{\mathrm{Ca}}$, induced robust CoMIC and eventual mitochondrial fragmentation in DN-Drp1-expressing neuron (Supplementary Fig. 13), although the effect of NS1619 may not be a result from mitoBK $_{\mathrm{Ca}}$ opening ${ }^{44}$. Collectively, these data indicate that
mitoBK $\mathrm{Ca}$ is involved in mitochondrial bulging and depolarization during CoMIC.

Cleavage of Opal synergistically regulates $\mathrm{CoMIC}$ with $\mathrm{Ca}^{2+}$. Recently, Opal has been reported as a coupler between mitochondrial morphology and transient mitochondrial depolarization during CoMIC ${ }^{24}$. Because we found that mitochondrial $\mathrm{Ca}^{2+}$ rise is required, but not sufficient for CoMIC (Fig. 5a), we wondered a possible role of Opal in CoMIC. First, we knocked down the expression of Opal with shRNA (shOpa1), and found that shOpal efficiently inhibits CoMIC without affecting DNDrp1-induced mitochondrial hyper-elongation (Fig. 8a,b and Supplementary Fig. 14). Although the probability of CoMIC was not affected, the total duration and frequency of CoMIC were 


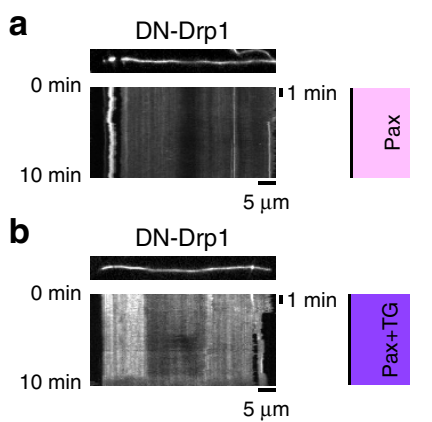

f

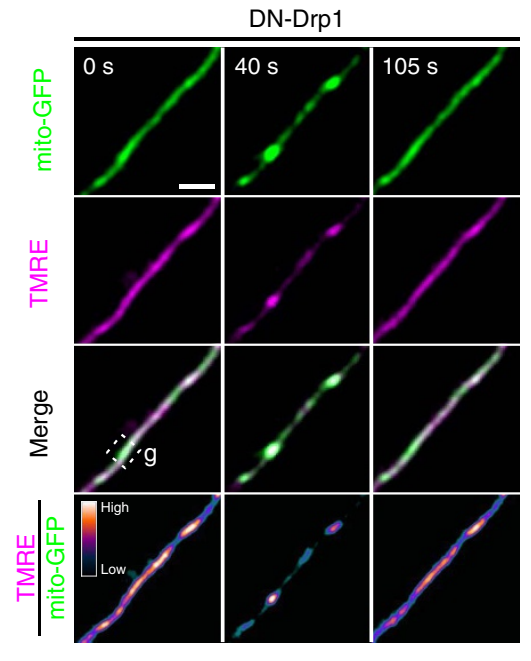

d

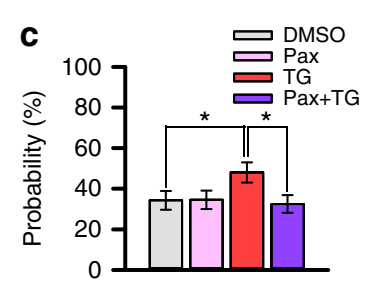

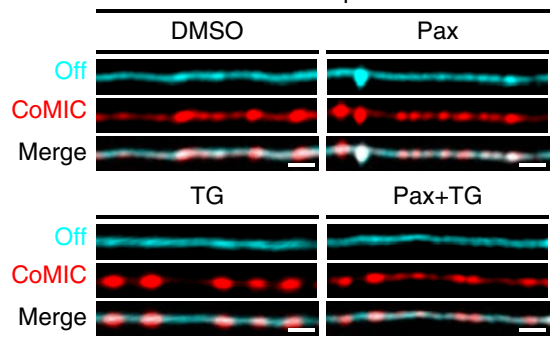

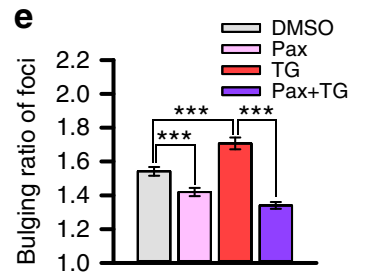

DN-Drp1+Pax

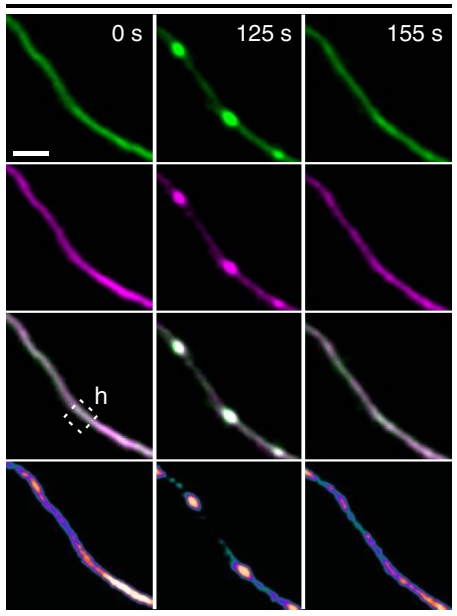

g

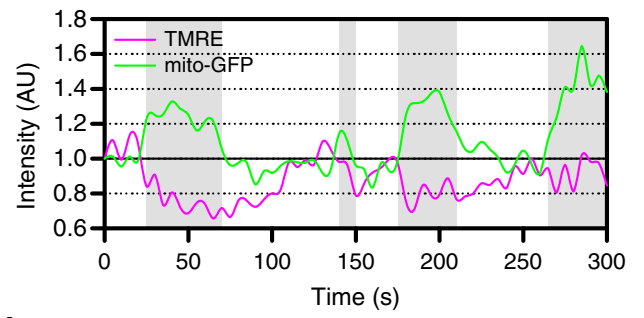

$\mathbf{h}$

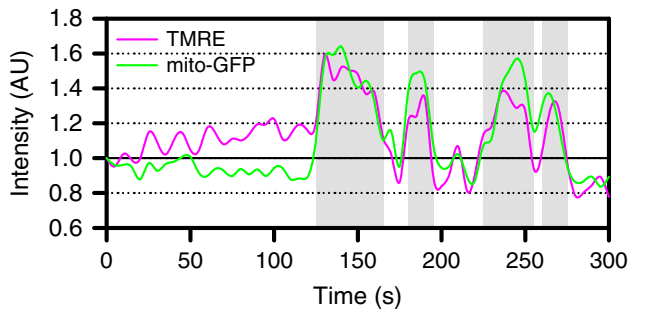

Figure 7 | Intra-mitochondrial $\mathbf{K}^{+}$influx is involved in mitochondrial bulging and depolarization during CoMIC. (a,b) Representative kymographies of DsRed-mito and patterns of CoMIC (right) in DN-Drp1-expressing neurons after treatment with paxilline (Pax, $10 \mu \mathrm{M})(\mathbf{a})$ and TG with 30 min pretreatment with $\mathrm{Pax}(\mathrm{Pax}+\mathrm{TG})$ for $1 \mathrm{~h}$ (b). (c) Probability of CoMIC in elongated mitochondria treated with DMSO (103 mitochondria), Pax (110 mitochondria), TG (100 mitochondria) and Pax + TG (117 mitochondria) $\left({ }^{\star} P<0.05\right.$ by Mann-Whitney rank sum test). Error bar indicate s.e. (d) Straightened images of DsRed-mito showing minimal (cyan signal) and maximal bulging (red signal). (e) Quantification of bulging ratio in foci stained with DsRed-mito ( ${ }^{\star \star} P<0.01$ by Mann-Whitney rank sum test). (f) Time-lapse images of mitochondria labelled by mito-GFP and TMRE in DN-Drp1expressing neurons without or with Pax treatment. Scale bar, $5 \mu \mathrm{m}$, and TMRE/mito-GFP is pseudo-coloured. (g,h) Temporal fluctuations of TMRE and mito-GFP in dotted box of $\mathbf{f}$. Gray boxes indicate CoMIC duration of mitochondria.

significantly decreased by shOpal (Fig. 8e). In addition, shOpa1 significantly reduced the TG-increased probability of CoMIC as well as total duration and average single duration (Fig. 8c-e). These data indicate that Opal is required for induction and potentiation of CoMIC. We further examined whether other mitochondrial fusion machinery, Mfn1 and Mfn2, is also involved in CoMIC. Single depletion of Mfn1 or Mfn2 by shRNA affected neither DN-Drp1-potentiated CoMIC nor mitochondrial morphology (Supplementary Fig. 6). Notably, double depletion of Mfn1 and Mfn2 strongly inhibited DN-Drp1-induced mitochondrial hyper-elongation, thereby reducing CoMIC, which occurs in only elongated mitochondria. However, elongated mitochondria also exhibited DN-Drp1-potentiated CoMIC, and it appears that mitochondrial fusion indirectly contributes to potentiation of CoMIC by elongating mitochondria.

It has been known that IMM bound long Opa1 (L-Opa1) is cleaved by Oma1, which senses mitochondrial depolarization, resulting in the accumulation of soluble short Opal (S-Opa1) in the intermembrane space ${ }^{45}$. Thus, we postulated that Omal is upstream regulator of Opal in CoMIC. Very similarly to shOpa1, depletion of Omal by shRNA significantly reduced, but not probability, the total and average single duration of CoMIC without modifying DN-Drp1-induced mitochondrial hyper- elongation (Fig. 8f,g and Supplementary Fig. 15). It indicates that Oma1-mediated cleavage of Opal may play a role in the regulation of CoMIC. Recently, it has been demonstrated that S-Opal can induce mitochondrial fragmentation and its GTPasedefective mutant localizes to the foci of constriction and division with Drp1 at the ER-mitochondria contact site ${ }^{46}$. Thus, we further examined the differential involvement of the S-Opal and L-Opa1 in CoMIC. We co-transfected neurons with S-Opal fusing intermembrane space-localizing signal of apoptosisinducing factor or L-Opal lacking cleavage sites with DN-Drp1 (Supplementary Fig. 16a). While S-Opal induced excessive mitochondrial fragmentation in neurons and 293T cells as shown in previous report ${ }^{46}$, its co-expression with DN-Drp1 did not affect mitochondrial hyper-elongation by DN-Drp1 (Supplementary Fig. 16b,c), indicating that S-Opa1-induced mitochondrial fragmentation requires Drp1. Interestingly, in neurons co-expressing S-Opa1 with DN-Drp1, a greater proportion of mitochondria exhibited CoMIC-like structures (Fig. 8i). Quantitative analyses showed that the probability, total duration and average single duration of CoMIC were dramatically increased by S-Opal (Fig. 8h,i,n), indicating that the S-Opa1 sufficiently promotes induction/potentiation of CoMIC. In contrast, L-Opal induced mitochondrial elongation in control neurons, but it did not affect in DN-Drp1-expressing 

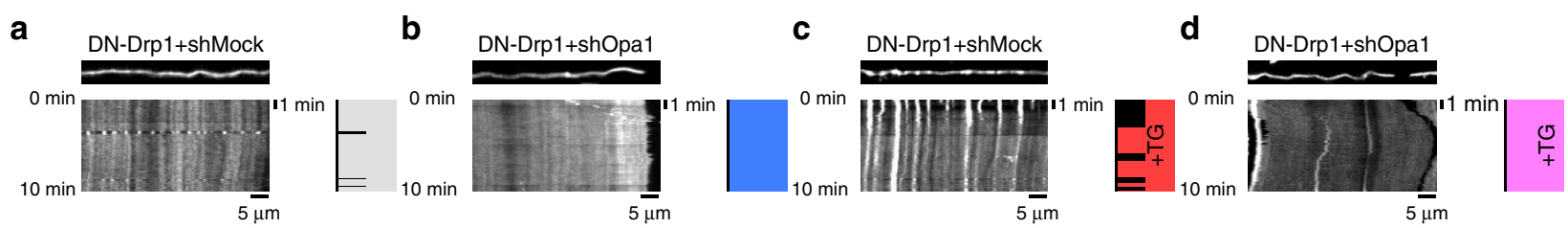

e
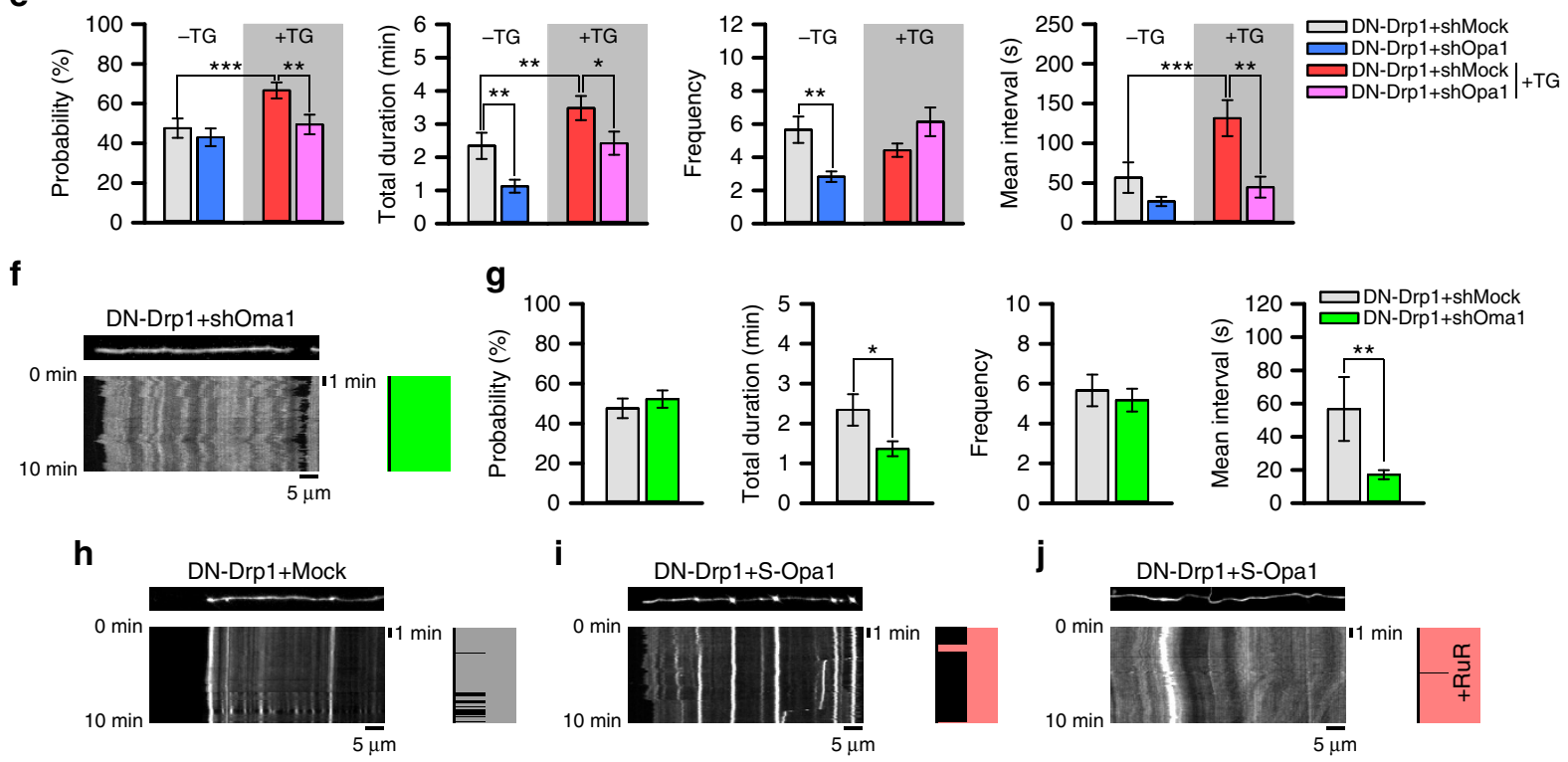

i
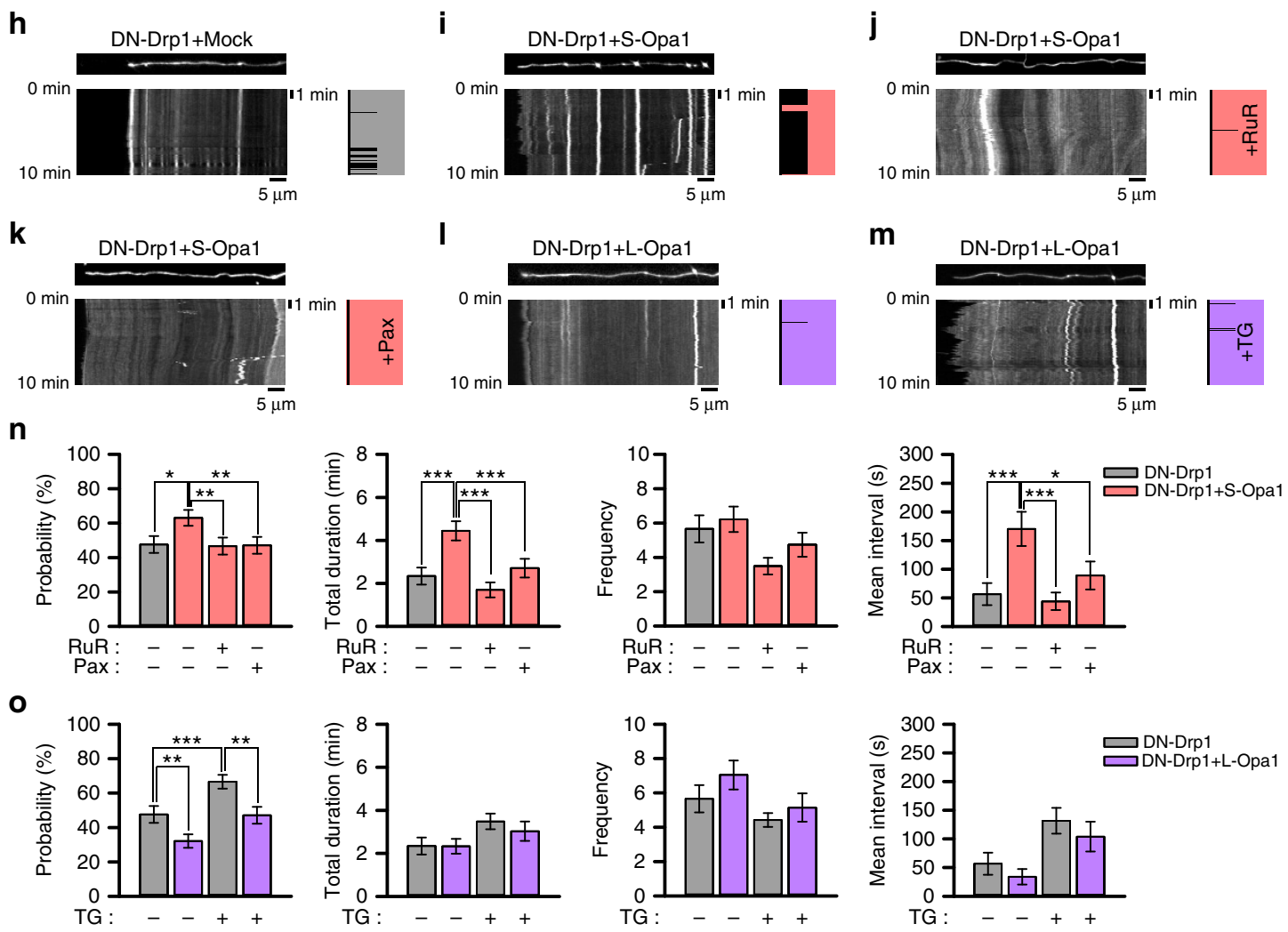

Figure 8 | Oma1-mediated cleavage of Opa1 regulates CoMIC. (a-d) Representative kymographies of DsRed-mito and patterns of CoMIC (right diagram) in DN-Drp1 + shMock- (a), DN-Drp1 + shOpa1- (b), TG-treated DN-Drp1 + shMock- (c) or TG-treated DN-Drp1 + shOpa1-expressing neurons (d). (e) Probability of CoMIC in elongated mitochondria from DN-Drp1+ shMock-, DN-Drp1+ shOpa1- (123 mitochondria), TG-treated DN-Drp1 + shMock- (138 mitochondria) and TG-treated DN-Drp1 + shOpa1-expressing neurons (105 mitochondria), and quantification of the total duration, frequency and average single duration of CoMIC $\left({ }^{\star \star} P<0.01,{ }^{\star \star} P<0.02\right.$ and ${ }^{\star} P<0.05$ by Mann-Whitney rank sum test). Error bar indicate s.e. (f) Representative kymography of DsRed-mito and patterns of CoMIC (right diagram) in DN-Drp1 + shOma1-expressing neuron. (g) Probability of CoMIC in elongated mitochondria from DN-Drp1 + shMock- and DN-Drp1 + shOma1-expressing neurons (134 mitochondria), and quantification of the total duration, frequency and average single duration of CoMIC ( ${ }^{\star \star} P<0.02$ and ${ }^{\star} P<0.05$ by Mann-Whitney rank sum test). (h-m) Representative kymographies of DsRed-mito and patterns of CoMIC (right diagram) in DNDrp1- (h), DN-Drp1 + S-Opa1- (i), ruthenium Red (RuR, $10 \mu \mathrm{M})$-treated DN-Drp1 + S-Opa1- (j), Pax-treated DN-Drp1 + S-Opa1- (k), DN-Drp1 + L-Opa1- (I) and TG-treated DN-Drp1 + L-Opa1-expressing neurons $(\mathbf{m})$. (n) Probability of CoMIC in elongated mitochondria from DN-Drp1- (105 mitochondria), DN-Drp1 + SOpa1- (111 mitochondria), RuR-treated DN-Drp1 + S-Opa1- (105 mitochondria) and Pax-treated DN-Drp1 + S-Opa1-expressing neurons (104 mitochondria), and quantification of the total duration, frequency and average single duration of CoMIC ( ${ }^{\star \star} P<0.01,{ }^{\star \star} P<0.02$ and ${ }^{\star} P<0.05$ by Mann-Whitney rank sum test). (o) Probability of CoMIC in elongated mitochondria from DN-Drp1-, TG-treated-DN-Drp1- (138 mitochondria), DN-Drp1 + L-OPA1- (140 mitochondria) and TGtreated DN-Drp1 + L-Opa1-expressing neurons (104 mitochondria), and quantification of the total duration, frequency and average single duration of CoMIC. $\left({ }^{\star \star \star} P<0.01\right.$ and ${ }^{\star \star} P<0.02$ by Mann-Whitney rank sum test). 
neuron (Supplementary Fig. 16b,c). Notably, L-Opal exhibited inhibitory effects on the induction of basal CoMIC and TGinduced CoMIC (Fig. 81,m,o), indicating inhibitory role of L-Opal in CoMIC.

Interestingly, the effect of S-Opal was efficiently blocked by ruthenium Red (RuR), an inhibitor of mitochondrial calcium uptake, or Pax (Fig. 8j,k,n). These data suggest that S-Opa1induced/potentiated CoMIC requires intra-mitochondrial influx of $\mathrm{Ca}^{2+} / \mathrm{K}^{+}$. Taken together, these data suggest that intramitochondrial influx of $\mathrm{Ca}^{2+} / \mathrm{K}^{+}$and cleavage of Opal are two interdependent pathways for the regulation of CoMIC.

Opa1 regulates OMM-IBM tethering during CoMIC. Finally, we explored how S-Opal regulates CoMIC. S-Opa1 is a component of mitochondrial contact site and intermembrane space bridging (MIB) complex, which play a role in cristae morphogenesis and mitochondrial inner boundary membrane (IBM)-OMM tethering, together with Mic60 (Mitofilin/Immt), Mic19 (ChchD3), Mic25 (ChchD6) and Sam50 (refs 47,48). Based on the rearrangement of OMM-IBM during CoMIC (Fig. 4), we postulated the involvement of Mic60 in the regulation of CoMIC by S-Opal. Remarkably, Mic60 depletion by shRNA significantly increased probability of CoMIC in DN-Drp1-expressing neurons (Fig. 9a,b and Supplementary Fig. 17a), although it did not affect DN-Drp1induced mitochondrial hyper-elongation (Supplementary Fig. 17b,c). Conversely, Mic60 overexpression strongly blocked the induction and potentiation of CoMIC in DN-Drp1-expressing neurons (Fig. 9c,d and Supplementary Fig. 17a,d,e). Quantitative analyses showed that the probability, total duration and mean interval of CoMIC are markedly decreased by Mic60 overexpression (Fig. 9g). Co-expression of Mic60 with S-Opa1 in DN-Drp1expressing neuron remarkably inhibited S-Opa1-induced/potentiated CoMIC (Fig. 9e-g), suggesting that Mic60 is inactivated by
S-Opal and the S-Opal-induced CoMIC can be reversed by overexpression of Mic60 (Fig. 10).

\section{Discussion}

In this study, we found that a subset of elongated mitochondria underwent transient and repetitive CoMIC, which was closely associated with subsequent mitochondrial division. So far, CoMIC-like events have been proposed to be associated with mitochondrial physiologies, including mitochondrial uncoupling, ROS production and oxidative phosphorylation ${ }^{22-24}$. In this study, we suggest that CoMIC is strongly associated with mitochondrial division, based on that (1) mitochondria undergoing CoMIC have higher probability for subsequent mitochondrial division; (2) mitochondrial division occurs at the CoMIC foci; (3) suppression of Drp1 strongly influences the extent of CoMIC; and (4) conditions blocking CoMIC suppressed mitochondrial division. Furthermore, by inhibiting mitochondrial division, we dissected intra-mitochondrial events mediating the CoMIC; (1) intra-mitochondrial $\mathrm{Ca}^{2}+$ flux triggering CoMIC, (2) mitoK $_{\mathrm{Ca}}$-mediated mitochondrial bulging and depolarization and (3) Omal-mediated accumulation of S-Opa1, which neutralizes Mic60-mediated OMM-IBM tethering (Fig. 10). Therefore, we propose that CoMIC is an intra-mitochondrial priming event for mitochondrial division.

Although we primarily focused on the mitochondria in neuronal processes, CoMIC was also found in multiple cell types including fibroblasts (mesoderm-derived cells) and HepG2 cells (endodermderived cells) as well as neurons (ectoderm-derived cells). Previous reports have also showed CoMIC-like mitochondrial constriction in other many mammalian cells under normal and pathologic conditions ${ }^{22-25,49-52}$. Furthermore, this event has also been observed in yeast $^{13}$, C. elegans ${ }^{14}$ and even plants ${ }^{53}$. Therefore, we suggest that the fundamental machinery triggering CoMIC may be

a
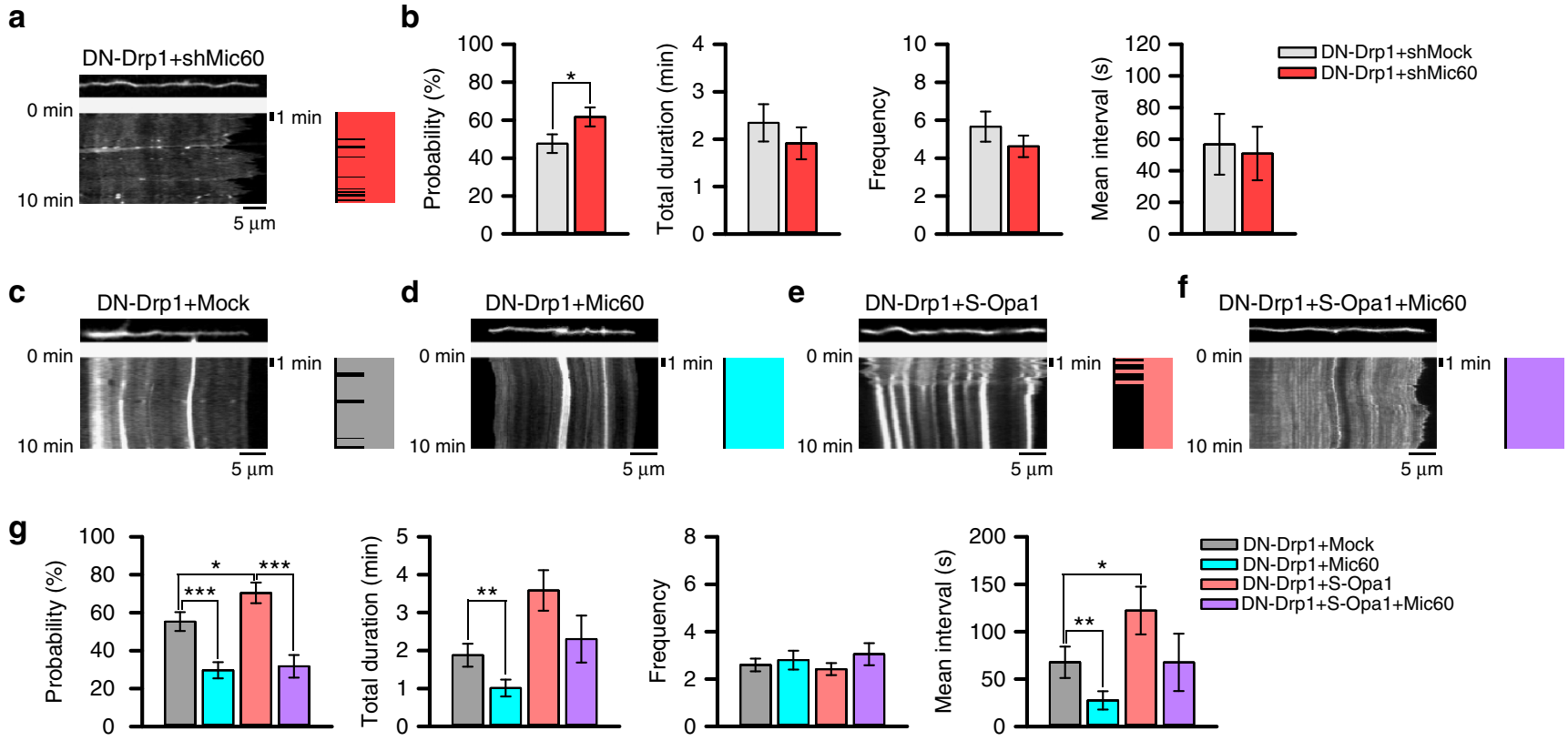

Figure 9 | Opa1 neutralizes Mic60-mediated tethering ОMM and IMM so regulating CoMIC. (a) Representative kymography of DsRed-mito and patterns of CoMIC (right diagram) in DN-Drp1+ shMic60-expressing neurons. (b) Probability of CoMIC in elongated mitochondria from DNDrp1 + shMock- and DN-Drp1 + shMic60-expressing neurons (94 mitochondria), and quantification of the total duration, frequency and average single duration of CoMIC ( ${ }^{\star} P<0.05$ by Mann-Whitney rank sum test). Error bar indicate s.e. (c-f) Representative kymographies of mito-GFP and patterns of CoMIC (right diagram) in DN-Drp1+Mock (c), DN-Drp1+Mic60- (d), DN-Drp1+S-Opa1- (e) and DN-Drp1+S-Opa1+ Mic60-expressing neurons (f). (g) Probability of CoMIC in elongated mitochondria from DN-Drp1-, DN-Drp1 + Mic60- (118 mitochondria), DN-Drp1 + S-Opa1- (71 mitochondria) and DNDrp1 + S-Opa1 + Mic60-expressing neurons (63 mitochondria), and quantification of the total duration, frequency and average single duration of CoMIC $\left({ }^{\star \star \star} P<0.01,{ }^{\star \star} P<0.02\right.$ and ${ }^{\star} P<0.05$ by Mann-Whitney rank sum test). 


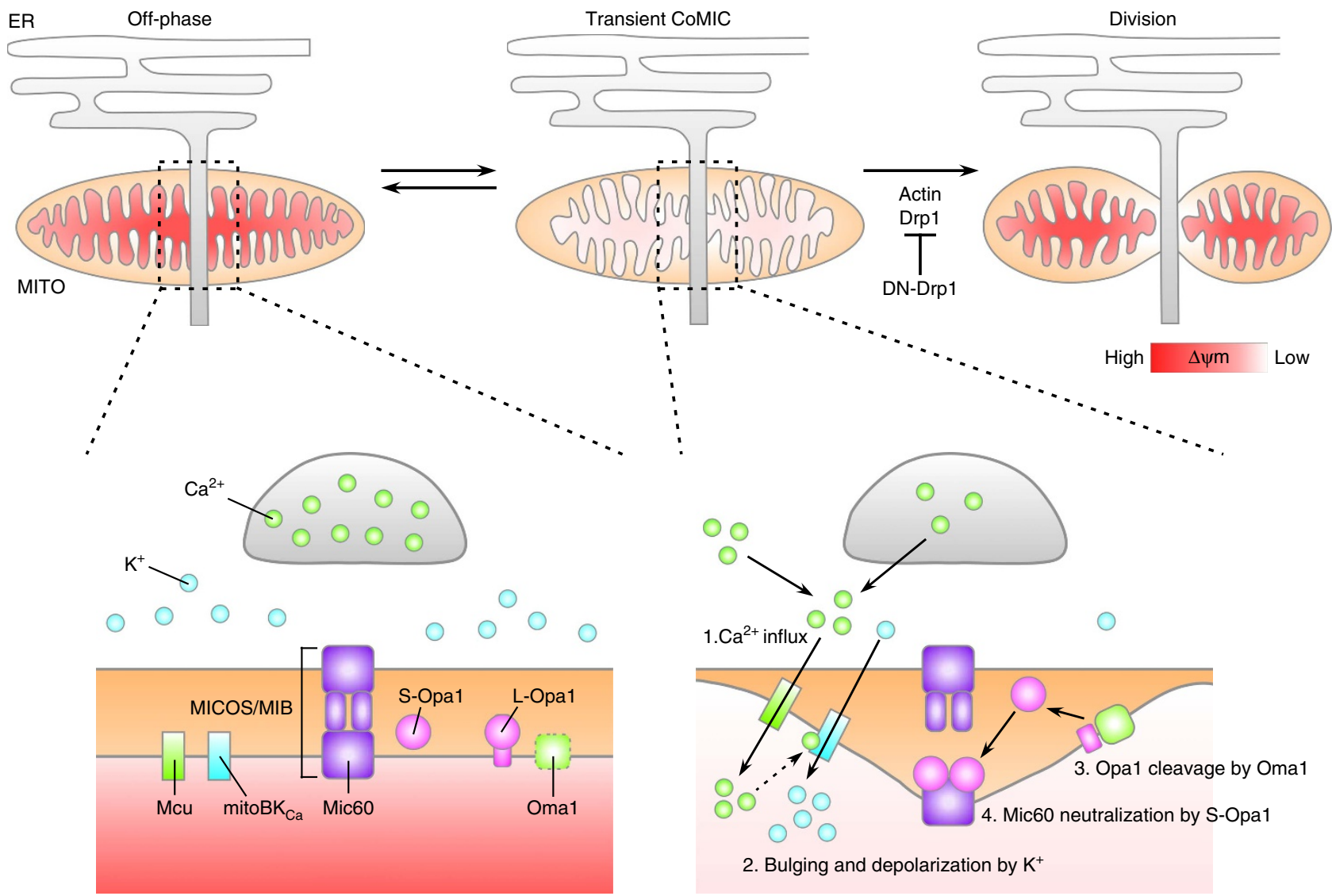

Figure 10 | Schematic model of CoMIC for mitochondrial division. Intra-mitochondrial $\mathrm{Ca}^{2}+$ flux triggers mitoBK $\mathrm{Ca}_{\mathrm{a}}$-mediated mitochondrial bulging and depolarization. Synergistically, stabilized Oma1 cleaves L-Opa1 resulting accumulation of S-Opa1, which neutralizes Mic60-mediated OMM-IBM tethering. This CoMIC contributes to Drp1-mediated mitochondrial division.

conserved and operating in most, if not all, eukaryotic cells. Mitochondrial influx of $\mathrm{Ca}^{2+}$ plays an essential role in the induction of CoMIC. Interestingly, $\mathrm{Ca}^{2}+$ also promotes cellular constriction in prokaryotic cells by enhancing FtsZ polymerization and further bundling of FtsZ polymers ${ }^{54}$. Although mammalian FtsZ ring does not exist, this implies that $\mathrm{Ca}^{2+}$-mediated CoMIC seems to be a fundamental and highly conserved machinery from prokaryotic to eukaryotic cells.

Controversial data reported that there was no detectable change in intra-mitochondrial $\mathrm{Ca}^{2}+$ concentration during a CoMIC-like event ${ }^{23}$. However, we used more sensitive genetic dye CEPIA3mt (ref. 37), and could detect rise on intra-mitochondrial $\mathrm{Ca}^{2}+$ during CoMIC. In addition, Ru360 and depletion of Mcu inhibited DN-Drp1-potentiated CoMIC. Considering that elongated mitochondria can more efficiently absorb $\mathrm{Ca}^{2}+$ than short mitochondria in neuronal cells ${ }^{55}$, mitochondrial hyperelongation by DN-Drp1 may amplify intra-mitochondrial $\mathrm{Ca}^{2+}$ signals. When Drp1 is expressed normally and mitochondria is endogenously elongated, CoMIC is very transient and the effect of intra-mitochondrial $\mathrm{Ca}^{2+}$ influx on CoMIC may be small enough to be virtually undetectable. Furthermore, we showed that blockade of intra-mitochondrial $\mathrm{Ca}^{2+}$ entry inhibits efficient mitochondrial division. Consistently with our data, previous pharmacological studies have shown that Ru360 inhibits mitochondrial fragmentation by $\mathrm{Ca}^{2}+$ released from the ER by p20, which is a cleaved form of BAP31 by caspase 8 during Fasmediated cell death ${ }^{39}$. Furthermore, inhibition of mitochondrial $\mathrm{Ca}^{2+}$ uptake by NIM811 and carbonyl cyanide-ptrifluoromethoxyphenyl-hydrazone, which are a mitochondrial permeability transition inhibitor and a mitochondrial uncoupler, also blocks mitochondrial fragmentation by NMDA-mediated entry of extracellular $\mathrm{Ca}^{2+}$ in neurons ${ }^{39,41,52,56}$. Conversely, cells with mutations in the Micul gene, which regulates Mcu activity, exhibit excessively fragmented mitochondria with increased intramitochondrial $\mathrm{Ca}^{2+}$ (ref. 57). These reports indicate that intramitochondrial $\mathrm{Ca}^{2+}$ is critical for efficient mitochondrial division. However, considering that mitochondrial division did not directly accompany intra-mitochondrial $\mathrm{Ca}^{2+}$ rise, $\mathrm{Ca}^{2+}$. mediated CoMIC is a prior event before mitochondrial division. In fact, intracellular $\mathrm{Ca}^{2+}$ activates cytosolic vesicle-forming machinery for mitochondrial division. Drp1 is upregulated by the $\mathrm{Ca}^{2+}$-dependent kinase CaMKI $\alpha$ following neuronal depolarization $^{58}$ or the $\mathrm{Ca}^{2+}$-dependent phosphatase calcineurin following mitochondrial damage and neuronal outgrowth ${ }^{59,60}$. In addition, $\mathrm{Ca}^{2+}$ can activate inverted formin2 , thereby promoting actin polymerization ${ }^{61}$. Therefore, $\mathrm{Ca}^{2+}$ mediates and coordinates both of cytosolic and intramitochondrial events for mitochondrial division. According to this model, a substantial increase in cytosolic $\mathrm{Ca}^{2}+$ can recruit Drp1 to the mitochondria to complete mitochondrial division, whereas CoMIC occurs when a lower level of $\mathrm{Ca}^{2+}$ is released from the ER and taken up into mitochondria.

Our data revealed that mitochondrial bulging during CoMIC is mediated by $\mathrm{K}^{+}$via mitoBK $\mathrm{Ca}$. Because the concentration of $\mathrm{K}^{+}$in the cytosol is higher than that in mitochondria, as the IMM is impermeable to potassium ions, mitochondrial influx of $\mathrm{K}^{+}$across the IMM requires specific channels, mitoBK $\mathrm{Ca}$ and $\operatorname{mitoK}_{\mathrm{ATP}}{ }^{19}$. Opening of these channels induces mitochondrial influx of $\mathrm{K}^{+}$, resulting in osmotic movement of water molecules into the mitochondrial matrix, thereby causing mitochondrial bulging or 
swelling. Potential role of intra-mitochondrial $\mathrm{K}^{+}$in mitochondrial volume and morphology has been elucidated. Intra-mitochondrial influx of $\mathrm{K}^{+}$promotes the formation of doughnut-like mitochondria during mitochondrial adaptation to conditions of hypoxiareoxygenation $^{62}$, and is also involved in formation of CoMIC-like structures by depletion of Letm1, which regulates homoeostasis of intra-mitochondrial $\mathrm{Ca}^{2+}$ and $\mathrm{K}^{+}$(ref. 63). Furthermore, inhibition of aquaporin by $\mathrm{HgCl}_{2}$ suppresses tunicamycin-induced CoMIC and further mitochondrial fragmentation ${ }^{64}$, indicating the involvement of osmosis in mitochondrial division. Notably, the $\mathrm{Ca}^{2+}$-responsive regulatory domain of $\operatorname{mitoBK}_{\mathrm{Ca}}$ is likely to face the mitochondrial matrix rather than the cytosol ${ }^{65}$, supporting the notion that intramitochondrial $\mathrm{Ca}^{2+}$, but not cytosolic $\mathrm{Ca}^{2+}$, mediates mitochondrial bulging. In addition, we found that DN-Drp1 enhances mitochondrial bulging during CoMIC, and it appears to result from increased $\mathrm{Ca}^{2+}$ uptake by mitochondrial hyperelongation ${ }^{55}$. Although previous report demonstrated that $\mathrm{H}^{+}$leak contributes to mitochondrial depolarization during $\mathrm{CoMIC}^{30,31}$, we found that inhibition of mitoBK $_{\mathrm{Ca}}$ efficiently suppresses mitochondrial depolarization during CoMIC. Recent study demonstrated the transient mitochondrial $\mathrm{pH}$ flash by bulky $\mathrm{H}^{+}$ leakage, which could be induced by intra-mitochondrial $\mathrm{Ca}^{2+} / \mathrm{K}^{+}$ influx ${ }^{66}$. Alternatively, the $\mathrm{H}^{+}$leak may contribute to recovery from CoMIC to off-phase, because efflux of $\mathrm{Ca}^{2+}$ and $\mathrm{K}^{+}$from the mitochondrial matrix are mainly mediated by a mitochondrial $\mathrm{Na}^{+} /$ $\mathrm{Ca}^{2+}$ exchanger and $\mathrm{K}^{+} / \mathrm{H}^{+}$exchanger driven by $\mathrm{H}^{+}$leak ${ }^{19}$. However, we also found that long exposure to carbonyl cyanide $m$-chlorophenyl hydrazone (CCCP), which disrupts the proton gradient and induces bulky $\mathrm{H}^{+}$leak, induces irreversible CoMIC with mitochondrial depolarization (Supplementary Fig. 18). Therefore, although we could not completely exclude that $\mathrm{H}^{+}$leak may mediate CoMIC, it appears that intra-mitochondrial $\mathrm{Ca}^{2}+/ \mathrm{K}^{+}$ influx mainly mediates morphological and physiological change during CoMIC.

Our data also suggested that Oma1-induced S-Opa1 induces and potentiates CoMIC. Recent studies have highlighted the role of S-Opal on mitochondrial division. Unlike L-Opa1, S-Opal sufficiently induced Drp1-dependent mitochondrial fragmentation in neurons, in agreement with a previous report ${ }^{46}$. Considering that potentiation of CoMIC in DN-Drp1expressing neurons was further strengthened by overexpression of S-Opa1, it is likely that S-Opal promotes CoMIC. However, shOpal did not influence the basal probability of CoMIC, although it efficiently suppressed the frequency and duration of CoMIC. Therefore, it is likely that S-Opal does not directly constrict the IMM, but is involved in the regulation of CoMIC. Based on our data with previous studies, S-Opal may have profission activity. However, S-Opal is required for proper mitochondrial fusion ${ }^{67}$, and in vitro IMM fusion can be promoted by S-Opal produced by i-AAA protease Yme1l under active oxidative phosphorylation and by Omal under mitochondrial depolarization ${ }^{46,68}$. This opposite effect of S-Opal could be mediated by disorganization of OMM-IBM contact via interplay between S-Opa1 and Mic60. The effect of S-Opa1 on the CoMIC was strongly modified by alterations of Mic60, which plays a role in tethering of OMM-IBM as core component of mitochondrial contact site and MIB complex ${ }^{47,48}$. Mic60 is evenly localized in $\mathrm{IMM}^{69}$, but S-Opa1 is preferentially localized in MIB complex and interacts with Mic60 (refs 47,48). Considering that S-Opal and L-Opal form oligomer ${ }^{70}$, this model may explain how L-Opa1 inhibits CoMIC: L-Opal may inhibit the S-Opa1and Mic60-mediated activation of CoMIC via neutralization of $\mathrm{S}$-Opal as a dominant-negative molecule. Therefore, we propose that S-Opal is involved in the disorganization of OMM-IBM, whereas L-Opal antagonizes this process, thereby contributing to the CoMIC and IMM reorganization.
It is likely that intra-mitochondrial $\mathrm{Ca}^{2+}{ }_{-} \mathrm{K}^{+}$and Oma1Opa1-Mic60 are two different axes for the control of CoMIC, and both are required for the efficient CoMIC. Therefore, inhibition of either cascades appear to inhibit CoMIC, while we do not entirely rule out the possibility that two cascades have significant interactions. For example, intra-mitochondrial influx of $\mathrm{Ca}^{2+}$ induces mild depolarization of mitochondria, leading to Oma1dependent cleavage of Opal (refs 40,46). On the other hand, Opal can also regulate intra-mitochondrial influx of $\mathrm{Ca}^{2+}$. In digitonin-permeabilized cells, depletion of Opa1 increases intramitochondrial influx of $\mathrm{Ca}^{2}+$ in spite of a dissipation of the mitochondrial membrane potential ${ }^{71}$, indicating that Opal plays a role in intra-mitochondrial structure for proper calcium uptake. Therefore, mutually dependent interaction between $\mathrm{Ca}^{2+}$ and Opal could be an important aspect explaining the cyclic nature of CoMIC, and the blockade of any part of this cascade can disrupt the CoMIC cycle.

\section{Methods}

Cell culture and gene transfection. Cortices were carefully dissected from Sprague-Dawley rats (Orient Bio and Koatech, Korea) on embryonic day 17 in prechilled Hank's buffered salt solution (Gibco) supplemented with 6\% glucose (Sigma) and 50 unit per ml penicillin-streptomycin (PS) (Gibco). Then, the samples were trypsinized and physically dissociated into single neurons. The dissociated neurons $\left(10^{5}\right.$ cells per $\left.\mathrm{cm}^{2}\right)$ were plated on glass coverslips, plates and dishes coated with poly-D-lysine (sigma), and incubated in neurobasal media (Gibco) containing 2\% B27 supplement (Gibco), $0.5 \mathrm{mM}$ L-glutamine (Gibco), $25 \mu \mathrm{M}$ L-glutamate (Sigma) and 50 unit per ml penicillin/streptomycin (Gibco) The neurons were incubated in $5 \% \mathrm{CO}_{2}$ at $37^{\circ} \mathrm{C}$. This experiment was carried out in strict accordance with the recommendations in the Guide for the Care and Use of Laboratory Animals of the Korea University Institutional Animal Care and Use Committee. The protocol was approved by the Committee on the Ethics of Animal Experiments of the Korea University (Permit Number: KUIACUC20110304-2). After 2 days in vitro (DIV2), we exchanged the medium for media containing no Lglutamate and maintained the cultured neurons in this medium. We transfected plasmids into cultured neurons on DIV2 or DIV8 using the Calphos mammalian transfection kit (Clontech) according to the manufacturer's protocol. After 24-48 h (DIV3-4 or DIV9-10), experiments were performed. Thapsigargin, Ru360 and latrunculin B were purchased from Calbiochem. Paxilline and CCCP were purchased from Abcam. Two-APB, ruthenium red and mito-TEMPO were purchased from Sigma. Drp1-knockout mouse embryonic fibroblasts were obtained from Dr Hiromi Sesaki ${ }^{17}$. 293T (ATCC CRL-3216), HeLa (ATCC CRM-CCL-2) and HepG2 (ATCC HB-8065) cells were incubated with Dulbecco's modified eagle medium (Gibco) supplemented with $10 \%$ foetal bovine serum (Gibco) and 50 unit per $\mathrm{ml}$ penicillin/streptomycin in $5 \% \mathrm{CO}_{2}$ at $37^{\circ} \mathrm{C}$, and gene transfection was performed using Lipofectamine 2000 (Invitrogen, USA) and the Calphos mammalian transfection kit according to the manufacturer's protocols. Experiments were performed $24 \mathrm{~h}$ after transfection.

DNA constructs. DsRed-mito and mito-GFP, which label the mitochondrial matrix, were purchased from Clontech. The PCR product of DN-Drp1 was obtained from pcDNA-Drpl (K38A) ${ }^{28}$ and inserted using the XhoI $\left(5^{\prime}\right)$ and EcoRI $\left(3^{\prime}\right)$ sites of pIRES2-GFP (Clontech) and pIRES-DsRed-mito, which was modified from pIRES2-GFP by exchanging GFP with DsRed-mito. Tom20-GFP was generated by insertion of a human Tom 20 PCR fragment into the XhoI $\left(5^{\prime}\right)$ and SalI $\left(3^{\prime}\right)$ sites of pEGFP-N1 (Clontech). GFP-Sec61b was generated by insertion of human Sec61b PCR fragment into the XhoI $\left(5^{\prime}\right)$ and EcoRI $\left(3^{\prime}\right)$ sites of pEYFP-C1 (Clontech). Via serial PCR, a flag-tagged S-Opal fragment was obtained by fusing the N-terminal signal peptide of apoptosis-inducing factor (amino acids 1-95) and the C-terminal of the human Opal isoform 1 cDNA (amino acids 202-969). A flagtagged L-Opal lacking proteolytic cleavage sites (amino acids 191-201) also was constructed by serial PCR. These DNA fragments were inserted into the BamHI $\left(5^{\prime}\right)$ and XhoI $\left(3^{\prime}\right)$ sites of pcDNA3.0 (Invitrogen, USA). In addition, myc-tagged Mic60 and Omal cDNAs were obtained by RT-PCR from RNA of rat cultured neuron, and were inserted into the BamHI $\left(5^{\prime}\right)$ and XhoI $\left(3^{\prime}\right)$ sites of pcDNA3.0. GFP-Mff (Addgene plasmid \# 49153) and mito-BFP (Addgene plasmid \# 49151) were gifts from Gia Voeltz. CEPIA3mt (Addgene plasmid \# 58219) and TwinkleGFP also were gifts from Masamitsu Iino and Johannes N. Spelbrink, respectively. The targeted sequences for shRNA were $5^{\prime}$-GAA GAG TGT AAC TGA TTC A-3' for rat Drp $1,5^{\prime}$-GCC AGA GAC AGA CAA TAC T- $3^{\prime}$ for rat $M c u, 5^{\prime}-$ GAT TGT GCC TGA CTT TAT A-3' for rat Opa1, 5' -CGA AAC CAG ATG AAC CTT T- $3^{\prime}$ for rat $M f n 1,5^{\prime}$-TGA GGA TGT TTG AGT TTC A- $3^{\prime}$ for rat $M f n 2,5^{\prime}$ GCC ATA AGA GAG GTC CGG A-3' for rat Omal and $5^{\prime}$-CTG AGA TTG CAG GTG AGA A-3' for rat Mic60. The shRNA constructs were generated using the pSuper.neo + GFP vector (OligoEngine) according to the manufacturer's protocol. 
Immunoblotting. Rat cortical neuron and 293T cell were collected in lysis buffer (125 mM Tris-Cl, 4\% SDS, pH 6.8). After SDS-PAGE and transfer into membrane, immunoblotting was performed using the following antibodies: anti-Opal (BD Transduction Laboratories, \#612606, 1:500), anti-Mcu (abcam, \#ab121499, 1:1,000), anti-myc (Santa Cruz Biotechnology, \#sc-40, 1:1,000) and anti-13-Actin (Santa Cruz Biotechnology, \#sc-47778, 1:2,000).

Live cell imaging and microscope image acquisition. Live cell imaging was mainly performed using an inverted fluorescence microscope (Carl Zeiss, Observer Z1) equipped with CoolLED (pE-2) as the light source, a definite focus module, a CCD camera (Photometrics, CoolSNAP fx), and a humidified chamber that maintained cells at $5 \% \mathrm{CO}_{2}$ and $37^{\circ} \mathrm{C}$. Primary neurons and other cell lines were incubated in the appropriate culture media during live cell imaging. A $\times 63$ oil-immersion objective lens (Carl Zeiss, EC Plan-Neofluar, 1.25 NA) was used for image acquisition, and timelapse images were obtained every $5 \mathrm{~s}$ for $10 \mathrm{~min}$ using the Metamorph (version 7.7.5) imaging program (Molecular Devices). In addition, we used a confocal inverted laserscanning microscope (Carl Zeiss, LSM7 LIVE) equipped with a humidified incubator that maintained cells in $5 \% \mathrm{CO}_{2}$ at $37^{\circ} \mathrm{C}$, and images were obtained with ZEN software (Carl Zeiss) with a $\times 63$ oil-immersion objective lens (Carl Zeiss, PlanApochromat, 1.4 NA). High-resolution live cell confocal images were obtained using an inverted laser-scanning microscope (Carl Zeiss, DE/LSM510 NLO) equipped with a humidified incubator that maintained cells in $5 \% \mathrm{CO}_{2}$ at $37^{\circ} \mathrm{C}$. A $\times 100$ oilimmersion objective lens (Carl Zeiss, EC Plan-Neofluar, $1.3 \mathrm{NA}$ ) was mainly used with $\times 3$ digital zoom for image acquisition. Confocal serial sectional images were obtained every $10 \mathrm{~s}$ for $10 \mathrm{~min}$ using ZEN software (Carl Zeiss). Each time-lapse image consists of four or five sections obtained with a centred Z-section mode. For synchronized imaging of dual channels, we used two mixed lasers, 488 and $536 \mathrm{~nm}$, for emission, and detected excitation using a splitter filter. To avoid excessive photo-damage caused by the LED or laser, we took time-lapse images from one slide within $1-1.5 \mathrm{~h}$ after drug treatment. The images were analyzed using the ImageJ program (USA National Institutes of Health). We linearly adjusted the intensity of the images, smoothed the images with 'Gaussian Blur' filtering and reconstructed three-dimensional images using the '3D viewer' module. The reconstructed images were recorded in $360^{\circ}$ rotation. For measurement of mitochondrial length, we first fixed primary neurons expressing DsRed-mito with 4\% paraformaldehyde in PBS, then stained their nuclei with $1 \mu \mathrm{g} \mathrm{ml}^{-1}$ Hoechst 33342. Then, we captured images using an upright fluorescent microscope (Carl Zeiss Axioskop2 Plus) with a CCD camera (Photometrics, CoolSNAP cf) using the Metamorph imaging programme. A $\times 40$ objective lens (Carl Zeiss, EC Plan-Neofluar, $0.75 \mathrm{NA}$ ) was used for image acquisition. In addition, we used a confocal inverted laser-scanning microscope (Carl Zeiss, LSM700). A $\times 40$ water-immersion objective lens (Carl Zeiss, LD C-Apochromat, 1.1 NA) was mainly used with $\times 3$ digital zoom for image acquisition. FRET images were acquired by confocal microscopy (Carl Zeiss, LSM700) using microscope filter combination for BFP and FRET: $405 \mathrm{~nm}$ excitation, $0-415 \mathrm{~nm}$ and $415-735 \mathrm{~nm}$ emission filters, respectively. Images were analysed using ImageJ software.

Kymographic and quantitative analyses of CoMIC. All images were modified with the ImageJ programme. For kymographic analysis, we modified time-lapse images of mitochondria in curved neuronal processes to straight images using the 'straighten' function. Then, the images were resliced to $y$ axis and Z-projected with the 'reslice' and 'Z-project' modules. We manually generated constriction spikes (right diagram of kymography). Each incidence of CoMIC was labelled as one spike, and the duration of the CoMIC was expressed by the thickness of the spike. We performed quantitative analyses, including the total duration, frequency and mean interval, of CoMIC from all mitochondria longer than $5 \mu \mathrm{m}$ in neuronal processes $\sim 200 \mu \mathrm{m}$ from the soma. To measure the bulging ratio of foci, we selected two mitochondrial images of off-phase and maximum bulging from more than 10 CoMIC-containing mitochondria, and measured the ratio of the diameter of each foci in the off-phase and bulging site.

Measurement of mitochondrial length. Mitochondrial images captured from all neuronal processes ( $200 \mu \mathrm{m}$ from the soma) were converted to binary images using the 'threshold' module in ImageJ. Then, the binary images were converted to images 1 pixel wide by the 'skeletonize' module, and mitochondrial length was assessed by the 'analyse particles' module.

Electron microscopy. The Drp1 knockdown viral constructs were transfected into 293gpg packaging cells using PEI (Polyscience, \#23966). After transfection, media were changed to the neurobasal media containing $2 \%$ B27 supplement, $0.5 \mathrm{mM} \mathrm{L}$ glutamine and 50 unit per $\mathrm{ml}$ penicillin/streptomycin. The supernatants containing viral particles were collected at 2-4 days after transfection, and infected primary cortical neurons. Drp1 knockdown cortical neurons were washed with $0.1 \mathrm{M}$ phosphate-buffered saline ( $\mathrm{pH} 7.4, \mathrm{PBS}$ ) and fixed with $10 \%$ paraformaldehyde, $2.5 \%$ glutaraldehyde in PBS at $4{ }^{\circ} \mathrm{C}$ for overnight. After fixation, cells were washed with PBS and post fixed with $1 \%$ osmium tetroxide for $90 \mathrm{~min}$. The fixed cells were dehydrated through ascending series of ethanol, and then embedded in Epon mixture. The embedding blocks were semi-thin sectioned $(200 \mathrm{~nm})$ using a Reichert-Jung Ultracut E ultramicrotome (Leica Microsystems), stained with Toluidine blue and trimmed for further observation. The trimmed sections were collected on 200-mesh cooper grid and stained with uranyl acetate and lead citrate. Observation of mitochondrial structure was performed using an H-7500 electron microscope (Hitachi) with $80 \mathrm{kV}$ acceleration voltage.

Statistical analysis. Statistical analyses and graph plotting were conducted using the Sigmaplot 12.5 software. Centre values and error bars of all graph indicate mean value and s.e., respectively. To measure the probability of CoMIC, we separated mitochondria into those that showed CoMIC and those that did not, and assigned them values of $100 \%$ or $0 \%$, respectively. Then, we averaged them and tested statistical significance with the Mann-Whitney rank sum test. To examine the statistical significance of the proportion of mitochondria undergoing subsequent division to that of mitochondria showing no or prior CoMIC, we generated contingency tables according to the presence of CoMIC and division. The contingency tables were tested with $\chi^{2}$-tests with Yates's correction for continuity. Similarly, contingency tables for mitochondria showing prior CoMIC among nondividing or dividing mitochondria were also tested with a $\chi^{2}$-test with Yates's correction for continuity. For statistical analysis of total CoMIC duration, frequency and mean interval and bulging ratio of the foci containing CoMIC, the Mann-Whitney rank sum test was used.

Data availability. The authors declare that all data supporting the findings of this study are available within the article and its Supplementary Information Files or from the corresponding author on reasonable request.

\section{References}

1. Youle, R. J. \& van der Bliek, A. M. Mitochondrial fission, fusion, and stress. Science 337, 1062-1065 (2012).

2. Kuroiwa, T. et al. Vesicle, mitochondrial, and plastid division machineries with emphasis on dynamin and electron-dense rings. Int. Rev. Cell Mol. Biol. 271, 97-152 (2008)

3. Chan, D. C. Fusion and fission: interlinked processes critical for mitochondrial health. Annu. Rev. Genet. 46, 265-287 (2012).

4. Loson, O. C., Song, Z., Chen, H. \& Chan, D. C. Fis1, Mff, MiD49, and MiD51 mediate Drp1 recruitment in mitochondrial fission. Mol. Biol. Cell 24, 659-667 (2013).

5. Ingerman, E. et al. Dnm1 forms spirals that are structurally tailored to fit mitochondria. J. Cell Biol. 170, 1021-1027 (2005).

6. Mears, J. A. et al. Conformational changes in Dnm1 support a contractile mechanism for mitochondrial fission. Nat. Struct. Mol. Biol. 18, 20-26 (2011).

7. Friedman, J. R. et al. ER tubules mark sites of mitochondrial division. Science 334, 358-362 (2011)

8. Korobova, F., Ramabhadran, V. \& Higgs, H. N. An actin-dependent step in mitochondrial fission mediated by the ER-associated formin INF2. Science 339, 464-467 (2013).

9. Lee, J. E., Westrate, L. M., Wu, H., Page, C. \& Voeltz, G. K. Multiple dynamin family members collaborate to drive mitochondrial division. Nature 540, 139-143 (2016).

10. Ferguson, S. M. \& De Camilli, P. Dynamin, a membrane-remodelling GTPase Nat. Rev. Mol. Cell Biol. 13, 75-88 (2012).

11. $\mathrm{Li}, \mathrm{H}$. et al. A Bcl-xL-Drp1 complex regulates synaptic vesicle membrane dynamics during endocytosis. Nat. Cell Biol. 15, 773-785 (2013).

12. Elde, N. C., Morgan, G., Winey, M., Sperling, L. \& Turkewitz, A. P. Elucidation of clathrin-mediated endocytosis in tetrahymena reveals an evolutionarily convergent recruitment of dynamin. PLoS Genet. 1, e52 (2005).

13. Nishida, K. et al. Dynamic recruitment of dynamin for final mitochondrial severance in a primitive red alga. Proc. Natl Acad. Sci. USA 100, 2146-2151 (2003).

14. Legesse-Miller, A., Massol, R. H. \& Kirchhausen, T. Constriction and Dnmlp recruitment are distinct processes in mitochondrial fission. Mol. Biol. Cell 14, 1953-1963 (2003).

15. Labrousse, A. M., Zappaterra, M. D., Rube, D. A. \& van der Bliek, A. M. C elegans dynamin-related protein DRP-1 controls severing of the mitochondrial outer membrane. Mol. Cell 4, 815-826 (1999).

16. Ishihara, N. et al. Mitochondrial fission factor Drp1 is essential for embryonic development and synapse formation in mice. Nat. Cell Biol. 11, 958-966 (2009).

17. Wakabayashi, J. et al. The dynamin-related GTPase Drp1 is required for embryonic and brain development in mice. J. Cell Biol. 186, 805-816 (2009).

18. Fujioka, H., Tandler, B. \& Hoppel, C. L. Mitochondrial division in rat cardiomyocytes: an electron microscope study. Anat. Rec. 295, 1455-1461 (2012).

19. Kaasik, A., Safiulina, D., Zharkovsky, A. \& Veksler, V. Regulation of mitochondrial matrix volume. Am. J. Physiol. Cell Physiol. 292, C157-C163 (2007).

20. Knott, A. B., Perkins, G., Schwarzenbacher, R. \& Bossy-Wetzel, E Mitochondrial fragmentation in neurodegeneration. Nat. Rev. Neurosci. 9, 505-518 (2008). 
21. Tondera, D. et al. SLP-2 is required for stress-induced mitochondrial hyperfusion. EMBO J. 28, 1589-1600 (2009).

22. MacVicar, T. D. \& Lane, J. D. Impaired OMA1-dependent cleavage of OPA1 and reduced DRP1 fission activity combine to prevent mitophagy in cells that are dependent on oxidative phosphorylation. J. Cell Sci. 127, 2313-2325 (2014).

23. Breckwoldt, M. O. et al. Multiparametric optical analysis of mitochondrial redox signals during neuronal physiology and pathology in vivo. Nat. Med. 20, 555-560 (2014)

24. Lee, H. \& Yoon, Y. Transient contraction of mitochondria induces depolarization through the inner membrane dynamin OPA1 protein. J. Biol. Chem. 289, 11862-11872 (2014)

25. Zhang, L. et al. Altered brain energetics induces mitochondrial fission arrest in Alzheimer's disease. Sci. Rep. 6, 18725 (2016).

26. Rizzuto, R., Brini, M., Pizzo, P., Murgia, M. \& Pozzan, T. Chimeric green fluorescent protein as a tool for visualizing subcellular organelles in living cells. Curr. Biol. 5, 635-642 (1995).

27. Cho, B. et al. CDK5-dependent inhibitory phosphorylation of Drp1 during neuronal maturation. Exp. Mol. Med. 46, e105 (2014).

28. Frank, S. et al. The role of dynamin-related protein 1 , a mediator of mitochondrial fission, in apoptosis. Dev. Cell 1, 515-525 (2001).

29. Yoon, Y., Pitts, K. R. \& McNiven, M. A. Mammalian dynamin-like protein DLP1 tubulates membranes. Mol. Biol. Cell 12, 2894-2905 (2001).

30. Lewis, S. C., Uchiyama, L. F. \& Nunnari, J. ER-mitochondria contacts couple mtDNA synthesis with mitochondrial division in human cells. Science 353, aaf5549 (2016).

31. Garrido, N. et al. Composition and dynamics of human mitochondrial nucleoids. Mol. Biol. Cell 14, 1583-1596 (2003).

32. Rajala, N., Gerhold, J. M., Martinsson, P., Klymov, A. \& Spelbrink, J. N. Replication factors transiently associate with mtDNA at the mitochondrial inner membrane to facilitate replication. Nucleic Acids Res. 42, 952-967 (2014).

33. Filadi, R. et al. Mitofusin 2 ablation increases endoplasmic reticulummitochondria coupling. Proc. Natl Acad. Sci. USA 112, E2174-E2181 (2015).

34. de Brito, O. M. \& Scorrano, L. Mitofusin 2 tethers endoplasmic reticulum to mitochondria. Nature 456, 605-610 (2008).

35. Chang, K. T., Niescier, R. F. \& Min, K. T. Mitochondrial matrix Ca2 + as an intrinsic signal regulating mitochondrial motility in axons. Proc. Natl Acad. Sci. USA 108, 15456-15461 (2011).

36. Brookes, P. S., Yoon, Y., Robotham, J. L., Anders, M. W. \& Sheu, S. S. Calcium, ATP, and ROS: a mitochondrial love-hate triangle. Am. J. Physiol. Cell Physiol. 287, C817-C833 (2004).

37. Suzuki, J. et al. Imaging intraorganellar $\mathrm{Ca} 2+$ at subcellular resolution using CEPIA. Nat. Commun. 5, 4153 (2014)

38. Xu, S. et al. Cadmium induced Drpl-dependent mitochondrial fragmentation by disturbing calcium homeostasis in its hepatotoxicity. Cell Death Dis. 4, e540 (2013).

39. Breckenridge, D. G., Stojanovic, M., Marcellus, R. C. \& Shore, G. C. Caspase cleavage product of BAP31 induces mitochondrial fission through endoplasmic reticulum calcium signals, enhancing cytochrome $\mathrm{c}$ release to the cytosol. J. Cell Biol. 160, 1115-1127 (2003).

40. Hom, J., Yu, T., Yoon, Y., Porter, G. \& Sheu, S. S. Regulation of mitochondrial fission by intracellular $\mathrm{Ca} 2+$ in rat ventricular myocytes. Biochim. Biophys. Acta 1797, 913-921 (2010).

41. Germain, M., Mathai, J. P., McBride, H. M. \& Shore, G. C. Endoplasmic reticulum BIK initiates DRP1-regulated remodelling of mitochondrial cristae during apoptosis. EMBO J. 24, 1546-1556 (2005).

42. Kann, O. \& Kovacs, R. Mitochondria and neuronal activity. Am. J. Physiol. Cell Physiol. 292, C641-C657 (2007).

43. Szewczyk, A., Jarmuszkiewicz, W. \& Kunz, W. S. Mitochondrial potassium channels. IUBMB Life 61, 134-143 (2009).

44. Gaspar, T. et al. Delayed neuronal preconditioning by NS1619 is independent of calcium activated potassium channels. J. Neurochem. 105, 1115-1128 (2008).

45. Head, B., Griparic, L., Amiri, M., Gandre-Babbe, S. \& van der Bliek, A. M. Inducible proteolytic inactivation of OPA1 mediated by the OMA1 protease in mammalian cells. J. Cell Biol. 187, 959-966 (2009).

46. Anand, R. et al. The i-AAA protease YME1L and OMA1 cleave OPA1 to balance mitochondrial fusion and fission. J. Cell Biol. 204, 919-929 (2014).

47. Ding, C. et al. Mitofilin and CHCHD6 physically interact with Sam50 to sustain cristae structure. Sci. Rep. 5, 16064 (2015)

48. Darshi, M. et al. ChChd3, an inner mitochondrial membrane protein, is essential for maintaining crista integrity and mitochondrial function. J. Biol. Chem. 286, 2918-2932 (2011).

49. Skulachev, V. P. et al. Thread-grain transition of mitochondrial reticulum as a step of mitoptosis and apoptosis. Mol. Cell Biochem. 256-257, 341-358 (2004).

50. Griparic, L., van der Wel, N. N., Orozco, I. J., Peters, P. J. \& van der Bliek, A. M. Loss of the intermembrane space protein Mgm1/OPA1 induces swelling and localized constrictions along the lengths of mitochondria. J. Biol. Chem. 279, 18792-18798 (2004)

51. Stavru, F., Palmer, A. E., Wang, C., Youle, R. J. \& Cossart, P. Atypical mitochondrial fission upon bacterial infection. Proc. Natl Acad. Sci. USA 110, 16003-16008 (2013).

52. Brustovetsky, T., Li, V. \& Brustovetsky, N. Stimulation of glutamate receptors in cultured hippocampal neurons causes $\mathrm{Ca} 2+$-dependent mitochondrial contraction. Cell Calcium 46, 18-29 (2009).

53. Jaipargas, E. A., Barton, K. A., Mathur, N. \& Mathur, J. Mitochondrial pleomorphy in plant cells is driven by contiguous ER dynamics. Front. Plant. Sci. 6, 783 (2015).

54. Beuria, T. K. et al. Glutamate-induced assembly of bacterial cell division protein FtsZ. J. Biol. Chem. 278, 3735-3741 (2003).

55. Saotome, M. et al. Bidirectional $\mathrm{Ca} 2+-$ dependent control of mitochondrial dynamics by the Miro GTPase. Proc. Natl Acad. Sci. USA 105, 20728-20733 (2008).

56. Hom, J. R., Gewandter, J. S., Michael, L., Sheu, S. S. \& Yoon, Y. Thapsigargin induces biphasic fragmentation of mitochondria through calciummediated mitochondrial fission and apoptosis. J. Cell Physiol. 212, 498-508 (2007).

57. Logan, C. V. et al. Loss-of-function mutations in MICU1 cause a brain and muscle disorder linked to primary alterations in mitochondrial calcium signaling. Nat. Genet. 46, 188-193 (2014).

58. Han, X. J. et al. CaM kinase I alpha-induced phosphorylation of Drp1 regulates mitochondrial morphology. J. Cell Biol. 182, 573-585 (2008).

59. Dickey, A. S. \& Strack, S. PKA/AKAP1 and PP2A/Bbeta2 regulate neuronal morphogenesis via Drp1 phosphorylation and mitochondrial bioenergetics. J. Neurosci. 31, 15716-15726 (2011).

60. Cereghetti, G. M. et al. Dephosphorylation by calcineurin regulates translocation of Drp1 to mitochondria. Proc. Natl Acad. Sci. USA 105, 15803-15808 (2008).

61. Shao, X., Li, Q., Mogilner, A., Bershadsky, A. D. \& Shivashankar, G. V. Mechanical stimulation induces formin-dependent assembly of a perinuclear actin rim. Proc. Natl Acad. Sci. USA 112, E2595-E2601 (2015).

62. Liu, X. \& Hajnoczky, G. Altered fusion dynamics underlie unique morphological changes in mitochondria during hypoxia-reoxygenation stress. Cell Death Differ. 18, 1561-1572 (2011).

63. Tamai, S. et al. Characterization of the mitochondrial protein LETM1, which maintains the mitochondrial tubular shapes and interacts with the AAAATPase BCS1L. J. Cell Sci. 121, 2588-2600 (2008).

64. Lee, J. S. et al. Aquaporin-assisted and ER-mediated mitochondrial fission: a hypothesis. Micron 47, 50-58 (2013).

65. $\mathrm{Xu}, \mathrm{W}$. et al. Cytoprotective role of $\mathrm{Ca} 2+-$ activated $\mathrm{K}+$ channels in the cardiac inner mitochondrial membrane. Science 298, 1029-1033 (2002).

66. Santo-Domingo, J., Giacomello, M., Poburko, D., Scorrano, L. \& Demaurex, N. OPA1 promotes $\mathrm{pH}$ flashes that spread between contiguous mitochondria without matrix protein exchange. EMBO J. 32, 1927-1940 (2013).

67. Song, Z., Chen, H., Fiket, M., Alexander, C. \& Chan, D. C. OPA1 processing controls mitochondrial fusion and is regulated by mRNA splicing, membrane potential, and Yme1L. J. Cell Biol. 178, 749-755 (2007).

68. Mishra, P., Carelli, V., Manfredi, G. \& Chan, D. C. Proteolytic cleavage of Opal stimulates mitochondrial inner membrane fusion and couples fusion to oxidative phosphorylation. Cell Metab. 19, 630-641 (2014).

69. Barrera, M., Koob, S., Dikov, D., Vogel, F. \& Reichert, A. S. OPA1 functionally interacts with MIC60 but is dispensable for crista junction formation. FEBS Lett. 590, 3309-3322 (2016).

70. Frezza, C. et al. OPA1 controls apoptotic cristae remodeling independently from mitochondrial fusion. Cell 126, 177-189 (2006).

71. Fulop, L., Szanda, G., Enyedi, B., Varnai, P. \& Spat, A. The effect of OPA1 on mitochondrial $\mathrm{Ca}(2)(+)$ signaling. PLoS ONE 6, e25199 (2011).

\section{Acknowledgements}

We thank Dr Min Kyung-Tai (Ulsan National Institute of Science and Technology, Korea) and Dr Kim Jaesang (Ewha Womans University, Korea) for helpful suggestions and comments on description of the manuscript. We also thank Ms. Na Ji Eun for her technical supports. This research was supported by the National Research Foundation (NRF-2015M3C7A1028790, NRF-2016M3A9C6937761 and NRF-2008-0057782) to W.S. and the DGIST Convergence Science Center Program (17-BD-04) to C.M. funded by the Ministry of Science, ICT \& Future Planning of Korea and by the NIH (GM089853) to H.S

\section{Author contributions}

B.C. designed the experiments, mainly performed the experiments, analysed the data and wrote manuscript. H.M.C., Y.J., H.D.K., Y.H.J. and M.S. performed additional experiments. C.M., H.K., K.K., H.S., I.J.R. and H.K. provided critical experimental materials and revived manuscript. W.S. supervised B.C., designed the experiments and wrote the paper. 


\section{Additional information}

Supplementary Information accompanies this paper at http://www.nature.com/ naturecommunications

Competing interests: The authors declare no competing financial interests.

Reprints and permission information is available online at http://npg.nature.com/ reprintsandpermissions/

How to cite this article: Cho, B. et al. Constriction of the mitochondrial inner compartment is a priming event for mitochondrial division. Nat. Commun. 8, 15754 doi: 10.1038/ncomms15754 (2017).

Publisher's note: Springer Nature remains neutral with regard to jurisdictional claims in published maps and institutional affiliations. (c) (i) Open Access This article is licensed under a Creative Commons Attribution 4.0 International License, which permits use, sharing, adaptation, distribution and reproduction in any medium or format, as long as you give appropriate credit to the original author(s) and the source, provide a link to the Creative Commons license, and indicate if changes were made. The images or other third party material in this article are included in the article's Creative Commons license, unless indicated otherwise in a credit line to the material. If material is not included in the article's Creative Commons license and your intended use is not permitted by statutory regulation or exceeds the permitted use, you will need to obtain permission directly from the copyright holder. To view a copy of this license, visit http://creativecommons.org/ licenses/by/4.0/

(C) The Author(s) 2017 